\title{
Collective Nostalgia: A Group-Level Emotion That Confers Unique Benefits on the Group
}

\author{
Tim Wildschut \\ University of Southampton \\ Sara Robertson \\ University of Oxford
}

\author{
Martin Bruder \\ University of Konstanz
}

\begin{abstract}
This research established collective nostalgia as a group-level emotion and ascertained the benefits it confers on the group. In Study 1, participants who reflected on a nostalgic event they had experienced together with ingroup members (collective nostalgia) evaluated the ingroup more positively and reported stronger intentions to approach (and not avoid) ingroup members than those who recalled a nostalgic event they had experienced individually (personal nostalgia), those who reflected on a lucky event they had experienced together with ingroup members (collective positive), and those who did not recall an event (no recall). In Study 2, collective (vs. personal) nostalgia strengthened behavioral intentions to support the ingroup more so than did recalling an ordinary collective (vs. personal) event. Increased collective self-esteem mediated this effect. In Study 3, collective nostalgia (compared with recall of an ordinary collective event) led participants to sacrifice money in order to punish a transgression perpetrated against an ingroup member. This effect of collective nostalgia was more pronounced when social identification was high (compared with low). Finally, in Study 4, collective nostalgia converged toward the group average (i.e., was socially shared) when participants thought of themselves in terms of their group membership. The findings underscore the viability of studying nostalgia at multiple levels of analysis and highlight the significance of collective nostalgia for understanding group-level attitudes, global action tendencies, specific behavioral intentions, and behavior.
\end{abstract}

Keywords: nostalgia, collective nostalgia, collective emotions, group processes, social identification

Nostalgia has suffered a bad reputation. Scholars have described it as an "immigrant psychosis" (Frost, 1938, p. 801), a "mentally repressive compulsive disorder" (Fodor, 1950, p. 25), "a regressive manifestation closely related to the issue of loss, grief, incomplete mourning, and, finally, depression" (Castelnuovo-Tedesco, 1980, p. 110), and as exemplifying "the many obvious nonfunctionalities of emotion" (Frijda, 1986, p. 475). Recent evidence shows that this negative view of nostalgia is undeserved. Adopting a prototype approach, according to which people's understanding of nostalgia becomes organized over time in terms of the representativeness of its features (Rosch, 1978), Hepper, Ritchie, Sedikides, and Wildschut (2012) found that laypersons conceptualize nostalgia as a predomi-

This article was published Online First September 22, 2014.

Tim Wildschut, School of Psychology, University of Southampton; Martin Bruder, Zukunftskolleg/Department of Psychology, University of Konstanz; Sara Robertson, Christ Church, University of Oxford; Wijnand A. P. van Tilburg and Constantine Sedikides, School of Psychology, University of Southampton.

This research was partially supported by a European Association of Social Psychology postdoctoral seedcorn grant and a University of Konstanz Zukunftskolleg mentorship grant to Martin Bruder.

Correspondence concerning this article should be addressed to Tim Wildschut, Centre for Research on Self and Identity, School of Psychology, University of Southampton, Southampton, SO17 1BJ, United Kingdom. E-mail: timw@soton.ac.uk nantly positive, past-oriented emotion. In nostalgic reverie, one remembers an event from one's past-typically a fond, personally meaningful memory. One often views the memory through rosetinted glasses, misses the remembered time or person(s), and may even long to return to the past. As a result, one typically feels sentimental, most often happy but with a tinge of longing. These lay conceptions of nostalgia are shared across cultures (Hepper, Wildschut, et al., 2014) and dovetail with formal dictionary definitions; The New Oxford Dictionary of English (Pearsall, 1998) defines nostalgia as "a sentimental longing or wistful affection for the past" ( $\mathrm{p}$. 1266). Furthermore, nostalgia confers important psychological benefits, including self-esteem (Hepper et al., 2012; Vess, Arndt, Routledge, Sedikides, \& Wildschut, 2012; Wildschut, Sedikides, Arndt, \& Routledge, 2006), social connectedness (Wildschut et al., 2006; Wildschut, Sedikides, Routledge, Arndt, \& Cordaro, 2010; Zhou, Sedikides, Wildschut, \& Gao, 2008), meaning in life (Routledge et al., 2011; Routledge, Wildschut, Sedikides, Arndt, \& Juhl, 2012; van Tilburg, Igou, \& Sedikides, 2013), optimism (Cheung et al., 2013), and approach motivation (Stephan et al., 2014).

Although this recent work has rehabilitated nostalgia, it has also focused exclusively on the individual level of analysis (personal nostalgia). That is, the work has documented the benefits that nostalgia confers on the individual, but it has not addressed the benefits that nostalgia might confer on the group. The key objective of the present research is to examine collective nostalgia and to shed light on its significance for understanding group processes. 
Intergroup emotions theory (IET; Mackie, Devos, \& Smith, 2000; Mackie \& Smith, 1998; Smith, 1993, 1999) postulates that when individuals think of themselves as part of a group, that group and events or objects related to it acquire emotional significance. Grouplevel emotions, according to this approach, include "a wide variety of positive and negative emotions - in fact, presumably any emotions that people can experience as individuals - that arise as a function of being a group member" (Smith, Seger, \& Mackie, 2007, p. 432). In keeping with this general perspective, we have defined collective nostalgia as the nostalgic reverie (as defined earlier) that is contingent upon thinking of oneself in terms of a particular social identity or as a member of a particular group (i.e., self-categorization at the collective level; Iyer \& Leach, 2009) and concerns events or objects related to it.

\section{Collective Nostalgia}

Content analysis of nostalgic autobiographical narratives has revealed that nostalgia is deeply imbued with sociality. These narratives typically feature the self in a major role but often in social context (e.g., with family, friends, or group members; Hepper et al., 2012; Holak \& Havlena, 1998; Wildschut et al., 2006). This prominent social component has led various scholars to speculate that nostalgia can solidify shared social identity (for a review, see Sedikides, Wildschut, Routledge, Arndt, \& Zhou, 2009). Volkan (1999) theorized that immigrants and refugees are especially likely to be nostalgic. He proposed that, in the context of an unfamiliar and potentially hostile host country, "linking objects" (e.g., songs associated with the homeland) and associated feelings of nostalgia become the core features of a collective identity. Kim (2010) reported that Korean immigrants to China expressed nostalgia about a collective way of living that they perceived to be threatened by the necessity to compete with the Han Chinese majority. Similarly, the "Red nostalgia" (i.e., nostalgia for the communist past) in some Eastern European countries may have key identity-affirming functions in a rapidly changing social and political environment (Blum, 2000; Gherghina \& Klymenko, 2012; Velikonja, 2009).

Collective nostalgia is also relevant at the organizational level of analysis. According to Gabriel (1993), "organisational nostalgia is not a marginal phenomenon, but a pervasive one" (p. 119). Employees who have worked for an organization for a sufficient period develop collective nostalgia that "binds them together" (Gabriel, 1993, p. 122). Milligan (2003) posited that among employees undergoing workplace relocation, nostalgic memories of the previous locations can serve as strong sources of "shared generational identities" (p. 399). Brown and Humphreys (2002) speculated that collective nostalgia might breed liking among group members, unify group members, and distinguish them from other groups. Therefore, collective nostalgia may be "key to the understanding of the dynamics of individual and organizational identity construction" (p. 141).

We proposed that nostalgia may also influence tangible decisions to support the collective. Recent research provides encouraging preliminary evidence that nostalgia facilitates prosocial decisions. This research has demonstrated that nostalgic (compared with nonnostalgic) charity appeals can increase charitable donations, volunteerism, and helping (Merchant, Ford, \& Rose, 2011; Zhou, Wildschut, Sedikides, Shi, \& Feng, 2012). In all, theoretical treatises on collective nostalgia suggest that it confers unique benefits on the group.

\section{Overview}

Our research had two major, novel objectives. The first objective was to test empirically the significance of collective nostalgia in terms of ingroup evaluation and global action tendencies to approach (and not avoid) ingroup members (Study 1), specific behavioral intentions to sacrifice time and effort to support the ingroup (Study 2), and actual behavior aimed at punishing a transgression against an ingroup member (Study 3).

The second objective was to examine whether collective nostalgia qualifies as a group-level emotion. Smith, Seger, and Mackie (2007) specified four criteria for conceptually and empirically identifying such group-level emotions: (a) group-level emotions can be differentiated from analogous individual-level emotions; (b) the assumption that emotions regulate human functioning and facilitate goal attainment (Frijda, 1986) implies that group-level emotions motivate and regulate attitudes and behavior in relation to social groups; (c) the experience and expression of group-level emotions are shaped by social identification; and (d) group-level emotions are socially shared or converge within a group. We explored whether collective nostalgia meets these criteria, focusing on the questions of whether collective nostalgia can be differentiated from personal nostalgia (Criterion 1; Studies 1 and 2); whether collective nostalgia motivates and regulates attitudes, global action tendencies, specific behavioral intentions, and actual behavior in relation to the ingroup (Criterion 2; Studies 1-3); whether the experience and expression of collective nostalgia are functions of social identification (Criterion 3; Study 3); and whether collective nostalgia is shared or converges within groups (Criterion 4 ; Study 4).

In the Preliminary Investigation and Studies $1-3$, we operationalized collective nostalgia by instructing undergraduate participants to recall a nostalgic memory of a shared experience with other ingroup members. Whereas the emphasis on shared experiences should be sufficient to render salient participants' social identity and encourage them to think of themselves as members of their group, we do not regard it as necessary. Accordingly, in Study 4, we operationalized collective nostalgia by directly instructing participants to think of themselves as a member of their national group and indicate to what extent they generally experienced nostalgia (Smith et al., 2007). ${ }^{1}$

\section{Preliminary Investigation}

Prior to conducting our primary studies, we carried out a preliminary investigation to establish the feasibility of studying nostalgia at the group level of analysis and form an impression of the

\footnotetext{
${ }^{1}$ Sample size varied across studies. This reflects practical constraints and variation in the size of the participant pools from which we recruited. In Studies 1-3, we recruited undergraduate students. For each of these studies, our pragmatic goal was to recruit as many participants as possible during the designated study period. In each case, the designated study period was a single academic semester. Studies $1(N=313)$ and $2(N=$ 171) both drew from a sizeable participant pool, but sample size was larger in the former study because it could be conducted in classroom settings, whereas the latter study was conducted in smaller laboratory environments. In Study $3(N=49)$, we recruited from a relatively small participant pool and were limited to collecting data from a single participant per experimental session. Finally, Study $4(N=108)$ was an online study completed via Amazon's Mechanical Turk (MTurk), for which we specified an a priori target of 100 participants. Variations in sample size do not reflect interim data-analysis or a flexible termination rule (Simmons, Nelson, \& Simonsohn, 2011).
} 
experiences that can constitute the basis of collective nostalgia. Forty-five University of Konstanz undergraduates (30 females; $M_{\text {age }}=23.62$ years, $S D_{\text {age }}=2.75$ ) provided narrative descriptions of a nostalgic event from their student life that they experienced together with other students. Two independent coders content analyzed these narratives $(\kappa=.83$; disagreements were resolved through discussion). Collective nostalgia related to extracurricular activities (e.g., celebrating, vacationing together; $n=27,60 \%$ ), standard curricular activities (e.g., attending lectures, preparing for exams; $n=13,29 \%$ ), and field trips (e.g., study visits to foreign countries, $n=5,11 \%$ ). Typical memories referred to student orientation at the start of university (e.g., meeting friends for the first time) and celebrating the end of exams. For example, one participant wrote (translated into English from the original German text), "We always think back to how, when, and in which bar we first met." Another participant wrote, "During the past summer, we spent a lot of time at the lake swimming, sunbathing, or simply hanging out, and one occasion, after we finished our exams, was especially great." Narrative accounts of collective nostalgia in this student sample focused on meaningful and endearing episodes in the history of the ingroup. The preliminary investigation thus provided encouraging evidence that nostalgia can be studied fruitfully at the group level of analysis.

\section{Study 1}

Two key criteria for identifying an emotion as group level are (a) that it can be differentiated from analogous individual-level emotions and (b) that it motivates and regulates attitudes and behavior in relation to social groups (Smith et al., 2007). In Study 1 , we began to explore whether collective nostalgia meets these criteria. To this end, we examined whether participants who recalled a nostalgic event shared with ingroup members (collectivenostalgia condition) subsequently reported more positive ingroup evaluation (e.g., described ingroup members as warm) and action tendencies (e.g., intended to spend time with ingroup members) than did participants who recalled a nostalgic event from their personal life as a unique individual (personal-nostalgia condition), participants who recalled a positive event shared with ingroup members (positive-collective-event condition), and participants who did not recall an autobiographical event (no-recall condition).

Inclusion of the personal-nostalgia condition is important because it allowed us to examine whether nostalgia per se could improve ingroup evaluation, irrespective of whether the nostalgic event is related to the ingroup. This is within the realm of possibility, given that personal nostalgia increases self-esteem (Hepper et al., 2012; Wildschut et al., 2006, 2012) and, in turn, self-esteem may be projected onto ingroups (Gramzow \& Gaertner, 2005; Gramzow, Gaertner, \& Sedikides, 2001). If nostalgia per se improves ingroup evaluation, this would undermine the utility of distinguishing between collective and personal nostalgia and would cast doubt on the status of collective nostalgia as a grouplevel emotion.

Inclusion of the positive-collective-event condition is crucial because it allows us to address the possibility that reflecting on any positive experience shared with ingroup members per se confers benefits on the group, irrespective of whether the recalled event is nostalgic. That is, by including this condition, we can examine whether the postulated beneficial effects of collective nostalgia are due merely to the positive affect (PA) it entails. After all, work on personal nostalgia shows that the content of nostalgic narratives is more positive than negative (Wildschut et al., 2006), and nostalgia typically (Hepper et al., 2012; Stephan, Sedikides, \& Wildschut, 2012; Verplanken, 2012; Wildschut et al., 2006, 2010; Zhou, Wildschut, Sedikides, Shi, et al., 2012, Study 1) but not always (Zhou, Wildschut, Sedikides, Shi, et al., 2012, Studies 2-4) increases PA. Although recent investigations continue to establish unique beneficial effects of personal nostalgia above and beyond PA (Cheung et al., 2013; Routledge et al., 2012; Stephan et al., 2012, 2014; Turner, Wildschut, \& Sedikides, 2012; Turner, Wildschut, Sedikides, \& Gheorgiu, 2013; van Tilburg et al., 2013; Zhou, Wildschut, Sedikides, Shi, et al., 2012), we needed to gauge the role of PA in the context of the current research on collective nostalgia. We did so by capitalizing on the principle that, whereas collective nostalgia often relates to positive experiences shared with ingroup members, not all positive shared experiences evoke collective nostalgia.

Finally, inclusion of the no-recall condition is informative, because it allowed us to examine whether collective nostalgia can elevate ingroup evaluation and approach action tendencies above participants' baseline levels. The inclusion of this pure control condition acquires significance in light of the fact that people's evaluation of and identification with meaningful social categories are notoriously resistant to experimental manipulation, presumably because the value of a naturally occurring group identity (such as membership in a student body) is anchored firmly in prior experience (Ellemers, Spears, \& Doosje, 2002; McCoy \& Major, 2003; Ramos, Correia, \& Alves, 2014). Thus, the no-recall condition provided a rigorous comparison level for assessing the impact of collective nostalgia.

\section{Method}

Participants and design. We randomly assigned 313 University of Southampton undergraduates (213 females, 96 males, and four individuals who did not report their gender; $M_{\text {age }}=19.86$ years, $S D_{\text {age }}=2.69$ ) to the four conditions of a one-way design (condition: collective nostalgia vs. personal nostalgia vs. collective positive event vs. no recall).

Procedure and materials. Instructions in the collectivenostalgia and personal-nostalgia conditions were based on an existing nostalgia induction that has been validated by prior research in the United Kingdom (Cheung et al., 2013; Hepper et al., 2012; Stephan et al., 2012; Wildschut et al., 2006, 2010) as well as Ireland (Van Tilburg et al., 2013), the United States (Routledge, Arndt, Sedikides, \& Wildschut, 2008; Routledge et al., 2011, 2012), and China (Zhou et al., 2008; Zhou, Wildschut, Sedikides, Chen, \& Vingerhoets, 2012; Zhou, Wildschut, Sedikides, Shi, et al., 2012). We instructed participants in the collective-nostalgia condition to bring to mind a nostalgic event from their student life that they had experienced together with other students at their university. Instructions in the personal-nostalgia condition prompted participants to recall a nostalgic event from their personal life as a unique individual.

In the collective-positive-event condition, we instructed participants to bring to mind a lucky event from their student life that they had experienced together with other students at their university. The New Oxford Dictionary of English (Pearsall, 1998) de- 
fines luck as "success or failure apparently brought by chance rather than through one's own actions" (p. 1098). Accordingly, we prompted participants in this condition to "think of a positive past event in your student life that you shared with other University of Southampton students that was brought on by chance rather than through your own actions." In this way, we aimed to induce recall of collective events that evoke PA but do not concern the type of meaningful and endearing episodes in the history of the ingroup that can elicit collective nostalgia (Preliminary Investigation). Simply put, our goal was to separate PA (high) and nostalgia (low). To be sure, we do not mean to suggest that collective nostalgia is the only positive group-level emotion that can confer benefits on the ingroup, but when the purpose is to separate collective nostalgia and PA, past shared experiences that are likely to elicit both are unsuitable benchmarks. Comparing the merits and demerits of various discrete positive group-level emotions is beyond the scope of the present research.

In the no-recall condition, participants completed the dependent variables without having first recalled an autobiographical event. Participants who were instructed to recall an event (i.e., all except those in the no-recall condition) summarized the event with five keywords. We present instructions for the collective-nostalgia, personal-nostalgia, and collective-positive-event conditions in the Appendix.

Next, participants completed a two-item assessment of state nostalgia (Wildschut et al., 2006): "Right now, I am having nostalgic feelings" and "I feel nostalgic at the moment" $(1=$ not at all, $7=$ very much; $\alpha=.97 ; M=3.47, S D=1.57)$. Following this assessment, they completed a two-item measure of PA ("Right now, I am in a positive mood" and "Right now, I feel good"; 1 = not at all, 7 = very much; Wildschut et al., 2006). We averaged these items to create a PA index $(\alpha=.94 ; M=5.10, S D=1.23)$. Participants then rated the extent to which students at their university were presently: "humorous," "warm," "flexible," "fun to be with," "dependable," and "trustworthy" $(1=$ not at all, $7=$ very much; adapted from Castano, Yzerbyt, Paladino, \& Sacchi, 2002). We averaged these items to create an ingroup-evaluation index $(\alpha=.86 ; M=4.77, S D=0.96)$. Finally, we assessed ingrouporiented action tendencies with six items (Turner et al., 2012). We instructed participants to "Please rate your reactions to University of Southampton students. What are your intentions toward University of Southampton students?" Items were "I want to talk to them," "find out more about them," "spend time with them," “ avoid them," "have nothing to do with them," and "keep them at a distance" ( 1 = strongly disagree, $7=$ strongly agree $)$. Separate analyses of the approach ("talk to them," "find out more about them," "spend time with them"; $\alpha=.82 ; M=4.78, S D=1.08$ ) and avoidance ("avoid them," "have nothing to do with them," "keep them at a distance"; $\alpha=.81 ; M=1.95, S D=0.94$ ) indices produced identical (i.e., mirror image) results. We therefore reverse-scored the avoidance items and averaged all six items to create an overall index of approach action tendencies in relation to the ingroup $(\alpha=.84 ; M=5.41, S D=0.88)$.

\section{Results}

We present means and standard deviations as a function of experimental condition in Table 1. With one exception (discussed later), participant gender did not qualify any of the significant results reported in this section and was therefore omitted from the final analyses. Degrees of freedom vary due to missing values.

State nostalgia. An analysis of variance (ANOVA) revealed a significant omnibus effect of condition on state nostalgia, $F(3$, $307)=30.71, p<.001, \eta^{2}=.23$. We followed this omnibus effect with a planned contrast between the two nostalgia conditions (collective-nostalgia and personal-nostalgia) and the two nonnostalgia conditions (collective-positive-event and no-recall). As intended, state nostalgia was significantly higher in the former than in the latter conditions, $F(1,307)=77.17, p<.001, \eta^{2}=.19$. Supplementary pairwise comparisons revealed that the collectivenostalgia and personal-nostalgia conditions both produced significantly $(p<.05)$ more nostalgia than the collective-positive-event and no-recall conditions. Engaging in either collective or personal nostalgia increased state nostalgia (compared with recalling a positive collective event and no recall). However, we expected that only recalling a collective nostalgic event would improve ingroup evaluation.

PA. An initial Condition $\times$ Gender ANOVA revealed a significant main effect for participant gender, indicating that women $(M=5.23, S D=1.20)$ reported higher levels of PA than did men $(M=4.82, S D=1.27), F(1,301)=5.03, p=.026, \eta^{2}=.02 . \mathrm{We}$ therefore retained participant gender in the final analysis, which additionally revealed a nonsignificant main effect of condition, $F(3,301)=1.06, p=.366, \eta^{2}=.01$, and a nonsignificant Condition $\times$ Gender interaction, $F(3,301)=0.55, p=.646, \eta^{2}=$ .01 . To assess whether the collective-nostalgia and collectivepositive-event conditions differed on PA, we compared them directly (notwithstanding the nonsignificant omnibus condition effect). As intended, the difference between these two conditions was numerically small $\left(\eta^{2}=.006\right)$ and not statistically significant, $F(1,301)=1.96, p=.167$. The collective-positive-event condition thus provides a meaningful benchmark to determine if recalling any shared positive experience per se confers benefits on the group, irrespective of whether the recalled event is nostalgic.

Ingroup evaluation. We obtained a significant omnibus effect of condition on ingroup evaluation, $F(3,308)=11.60, p<$

Table 1

Means (Standard Deviations) as a Function of Experimental Condition in Study 1

\begin{tabular}{lcccc}
\hline \multicolumn{1}{c}{ Measure } & $\begin{array}{c}\text { Collective } \\
\text { nostalgia }\end{array}$ & $\begin{array}{c}\text { Personal } \\
\text { nostalgia }\end{array}$ & $\begin{array}{c}\text { Collective } \\
\text { positive event }\end{array}$ & No recall \\
\hline State nostalgia & $4.10(1.34)$ & $4.15(1.43)$ & $3.14(1.45)$ & $2.39(1.33)$ \\
State positive affect & $5.46(1.16)$ & $5.01(1.21)$ & $5.04(1.33)$ & $4.87(1.20)$ \\
Ingroup evaluation & $5.25(0.83)$ & $4.59(0.96)$ & $4.72(0.98)$ & $4.51(0.90)$ \\
Approach action tendencies & $5.74(0.95)$ & $5.15(0.79)$ & $5.34(0.82)$ & $5.39(0.84)$ \\
\hline
\end{tabular}


$.001, \eta^{2}=.10$. We partitioned this omnibus effect by first testing a planned contrast between the collective-nostalgia condition and the three control conditions (personal-nostalgia, collectivepositive-event, and no-recall conditions). We then tested for significant differences among the three control conditions. The focal contrast revealed that ingroup evaluation was significantly more positive in the collective-nostalgia condition than in the pooled control conditions, $F(1,308)=31.84, p<.001, \eta^{2}=.09$. There were no significant differences among the three control conditions, $F(2,308)=1.18, p=.308, \eta^{2}=.007$. Supplementary pairwise comparisons indicated that ingroup evaluation was significantly more positive in the collective-nostalgia condition than in any of the three control conditions. In sum, collective nostalgia (compared with the three control conditions) improved ingroup evaluations.

Approach action tendencies. Results revealed a significant omnibus effect of condition on approach action tendencies, $F(3$, $307)=6.79, p<.001, \eta^{2}=.06$. We again partitioned this omnibus effect by first testing a planned contrast between the collective-nostalgia condition and the three control conditions and then testing for significant differences among the three control conditions. The focal contrast revealed that motivation to approach (and not avoid) ingroup members was stronger in the collectivenostalgia condition than in the pooled control conditions, $F(1$, $307)=16.81, p<.001, \eta^{2}=.05$. There were no significant differences among the three control conditions, $F(2,307)=1.69$, $p=.186, \eta^{2}=.01$. Supplementary pairwise comparisons showed that approach action tendencies were significantly stronger in the collective-nostalgia condition than in any of the three control conditions. In sum, collective nostalgia (compared with the three control conditions) strengthened motivation to approach (and not avoid) ingroup members.

Controlling for PA. The absence of a significant omnibus effect of condition on PA (see earlier section) suggests that PA cannot fully account for the beneficial effect of collective nostalgia (compared with the three control conditions) on ingroup evaluation and approach action tendencies. To confirm this, we controlled for PA in analyses of covariance (ANCOVAs). PA was significantly associated with both ingroup evaluation, $B=0.25, S E=0.04, F(1,306)=39.84, p<.001, \eta^{2}=$ .10 , and approach action tendencies, $B=0.21, S E=0.04, F(1$, $306)=32.60, p<.001, \eta^{2}=.09$. This underscores the importance of controlling for PA. However, the focal contrast comparing the collective-nostalgia condition with the combined control conditions remained significant for ingroup evaluation, $F(1,306)=22.57, p<.001, \eta^{2}=.06$, and for approach action tendencies, $F(1,306)=10.48, p=.001, \eta^{2}=.03$. As before, there were no significant differences among the three control conditions for either ingroup evaluation, $F(2,306)=0.79, p=$ $.453, \eta^{2}=.004$, or for approach action tendencies, $F(2,306)=$ $2.25, p=.107, \eta^{2}=.01$. Supplementary pairwise comparison revealed that when controlling for PA, scores on ingroup evaluation and approach action tendencies remained significantly higher in the collective-nostalgia condition than in any of the three control conditions. (The difference between the collective-nostalgia and no-recall conditions on approach action tendencies became marginal, $p=.093$ ).

\section{Discussion}

Study 1 demonstrated that collective nostalgia strengthened positive ingroup evaluation and promoted action tendencies to approach (and not avoid) ingroup members compared with three pertinent control conditions. We found no significant differences between the three control conditions. The contrast between the collective-nostalgia and personal-nostalgia condition provides strong support for conceptualizing collective nostalgia as a grouplevel emotion on the basis of Criterion 1: collective nostalgia can be differentiated from the analogous individual-level emotion. The comparison between the collective-nostalgia and collectivepositive-event condition reveals that collective nostalgia bestows unique benefits on the group, above and beyond those conferred by recalling positive shared experiences per se. This finding militates against the possibility that the beneficial effects of collective nostalgia are due to the PA it entails and extends mounting evidence for the unique beneficial effects of personal nostalgia above and beyond PA (Cheung et al., 2013; Routledge et al., 2012; Stephan et al., 2012, 2014; Turner et al., 2012, 2013; Van Tilburg et al., 2013; Zhou, Wildschut, Sedikides, Shi, et al., 2012). The difference between the collective-nostalgia and no-recall condition shows that collective nostalgia elevates ingroup evaluation and approach action tendencies above baseline levels. Jointly, these findings indicate that collective nostalgia is a group-level emotion on the basis of Criterion 2: it motivates and regulates attitudes and action tendencies in relation to social groups. Our aim in Study 2 was to extend this empirical foundation and test its robustness.

\section{Study 2}

Study 2 extended Study 1 in three ways. First, in Study 1, we assessed global action tendencies to approach (and not avoid) ingroup members. By focusing on action tendencies rather than concrete behaviors, we followed in the footsteps of previous studies on group-level emotions (Mackie et al., 2000; Smith et al., 2007). Maintaining such consistency with prior research is crucial, because it facilitates the integration of novel findings into the wider literature and contributes toward a comprehensive taxonomy of group-level emotions in terms of action tendencies. Nevertheless, a legitimate concern is that global action tendencies may not always be strongly linked to specific group-related behaviors ( $\mathrm{Fa}-$ zio \& Towles-Schwen, 1999; Mackie et al., 2000). There are several reasons for this, an important one being that actual behaviors are more constrained by situational factors than are global action tendencies. We began to address this concern in Study 2 by assessing participants' specific behavioral intentions to support the ingroup (henceforth, support intentions). To be precise, we asked participants how many hours they would be willing to invest in a publicity campaign to support their university. Numerous studies have shown that specific behaviors can be predicted with considerable accuracy from intentions to engage in the behaviors under consideration (Ajzen \& Fishbein, 2005). Thus, by assessing support intentions, in Study 2, we moved a step closer to the ultimate goal of better understanding group-related behavior. Given that investing time and effort on behalf of the ingroup implies personal costs, the present assessment of support intentions should also be less susceptible to self-presentational concerns or experimental demand than the Study 1 assessment of global action tendencies vis-à-vis the ingroup. 
Second, we examined possible mediating mechanisms linking collective nostalgia with behavioral intentions in relation to the ingroup. Luhtanen and Crocker (1992) proposed that just as individuals vary in evaluations of their personal identity (i.e., personal self-esteem), individuals also vary in evaluations of their social or collective identity (i.e., collective self-esteem, or CSE). Theoretical treatises (Brown \& Humphreys, 2002; Gabriel, 1993; Sedikides et al., 2009; Volkan, 1999) suggest that collective nostalgia fosters positive CSE. In turn, positive CSE has been found to predict tangible expressions of group and organizational commitment such as organizational citizenship behavior, group loyalty, and reduced turnover (Blader \& Tyler, 2009; Ellemers, Kortekaas, \& Ouwerkerk, 1999; Fuller et al., 2006; de Moura, Abrams, Retter, Gunnarsdottir, \& Ando, 2009). In light of these theoretical and empirical contributions, we focused our attention on the mediational role of CSE.

We assessed CSE with the Collective Self-Esteem Scale (CSES; Luhtanen \& Crocker, 1992). The CSES assesses individuals' evaluation of their social identity and the groups to which they belong (university community, in the present case). The CSES comprises four four-item subscales or facets: Importance to Identity (henceforth, Identity) assesses the extent to which ingroup membership is important to the individual's self-concept; Private CSE assesses the individual's own judgments of whether the ingroup is a worthwhile entity; Public CSE assesses perceived judgments of the ingroup by outsiders; and Membership CSE (henceforth, Membership) assesses the extent to which the individual feels like a worthy member of the ingroup. Factor-analytic evidence supports a hierarchical model that includes a general CSE second-order factor, which subsumes four first-order factors representing the CSES facets (Luhtanen \& Crocker, 1992). We therefore first conducted analyses of the CSES total score. We followed this with analyses of the four facets to achieve a more fine-grained understanding of the processes linking collective nostalgia to support intentions.

Third, in Study 1, we examined the possibility that reflecting on any positive shared experiences per se confers benefits on the group, irrespective of whether the recalled event is nostalgic. We did so by comparing the collective-nostalgia condition to a collective-positive event condition and by statistically controlling for self-reported PA in an ANCOVA. Neither analysis supported the idea that the beneficial effects of collective nostalgia are due merely to the PA it entails. Nevertheless, our approach had two limitations. One limitation is that we instructed participants in the collective-positive-past condition to recall a lucky event that they had experienced together with other students. These instructions may have led participants to recall events that were not only positive but also relatively rare or unrepresentative and consequently less relevant to participants' relation to the ingroup. Another limitation is that we assessed diffuse (as opposed to discrete) PA via self-report only.

In Study 2, we addressed the former limitation by including a condition in which we instructed participants to recall a normal event shared with ingroup members. To be precise, we manipulated independently whether participants reflected on a nostalgic or ordinary event from their past (event reflection) and whether the experience was shared or not shared with other ingroup members (context). We addressed the latter limitation by assessing PA with an unobtrusive method that did not rely on self-report. Specifically, we content-analyzed participant-generated narratives with the Linguistic Inquiry and Word Count (LIWC) software program (Pennebaker, Chung, Ireland, Gonzalez, \& Booth, 2007). LIWC offers a validated method for measuring verbal expression of emotion (Kahn, Tobin, Massey, \& Anderson, 2007). We used the program to calculate the percentage of discrete positive (LIWCPA; e.g., "happy," "love," "joy") and negative (LIWC-NA; e.g., "angry," "sad," "pain") emotion words. By taking these steps, we were able to examine whether collective (compared with personal) nostalgia confers unique benefits on the ingroup, above and beyond those conferred by reflecting on an ordinary collective (compared with personal) experience, controlling unobtrusively for the relative frequency of discrete positive and negative emotions in participants' narratives.

\section{Hypotheses}

We hypothesized that engaging in collective (compared with personal) nostalgia would strengthen support intentions more so than would recalling an ordinary collective (compared with personal) event. That is, collective (compared with personal) nostalgia would bestow unique benefits on the ingroup, above and beyond those bestowed by recalling an ordinary collective (compared with personal) event. We further hypothesized that the unique beneficial effect of collective (compared with personal) nostalgia on support intentions would be mediated by CSE. Finally, we predicted that this mediational model would receive support even when the emotional valence of the recalled event (as coded by LIWC) was controlled.

\section{Method}

Participants and design. We randomly assigned 171 University of Southampton undergraduates (122 females, 49 males; $M_{\text {age }}=21.22$ years, $\left.S D_{\text {age }}=4.53\right)$ to the conditions of a 2 (event reflection: nostalgic, ordinary) $\times 2$ (context: collective, personal) between-subjects design.

Procedure and materials. Participants were instructed to reflect upon an event from their past. Instructions in the two nostalgic-event conditions were the same as in Study 1. That is, we asked participants to bring to mind a nostalgic event from their student life that they experienced together with other students (collective-nostalgia condition) or from their personal life as a unique individual (personal-nostalgia condition). Instructions in the collective-ordinary and personal-ordinary conditions were based on an extensively validated protocol (Hepper et al., 2012; Routledge et al., 2011; Stephan et al., 2012; Zhou et al., 2008). We asked participants in the collective-ordinary condition to bring to mind an ordinary event from their student life that they experienced together with other students. Instructions in the personalordinary condition prompted participants to recall an ordinary event from their personal life as a unique individual. Prior research has established that recalling an ordinary autobiographical event gives rise to considerably more positive than negative affect (Wildschut et al., 2010, Study 4). This is reassuring, because it indicates that recall of ordinary experiences provides a suitable baseline for assessing the effects of nostalgic recall.

We asked participants to write a description of the recalled event and summarize their narrative with five keywords. Upon detailed inspection, we found that none of the narratives in the personal- 
context conditions pertained to participants' university affiliation. As a formal check on the context manipulation, we used two items ( $\alpha=$.75) adapted from Gaertner, Sedikides, and Graetz (1999). The response scales were anchored as follows (note that lower ratings indicate salience of group membership): $1=I$ am very similar to other University of Southampton students, $7=$ I am a unique individual; and $1=$ My personality attributes are quite similar to the attributes of other University of Southampton students, $7=$ My personality attributes are totally unique. As intended, a 2 (event reflection: nostalgic, ordinary) $\times 2$ (context: collective, personal) ANOVA revealed a context main effect only, $F(1,167)=5.38, p=.022, \eta^{2}=.03$. This supports our assumption that recalling events experienced together with other students (compared with events from their personal life as a unique individual) made salient participants' group membership (Table 2).

Next, participants completed the two-item measure of state nostalgia (see Study $1 ; 1=$ not at all, $7=$ very much; $\alpha=.93$; $M=4.31, S D=1.61)$. This was followed by the CSES $(1=$ not at all, $7=$ very much). We averaged items to calculate a CSES total score $(\alpha=.81 ; M=5.10, S D=0.67)$ as well as separate scores for the four facets: Identity (e.g., "Being a member of the social group of students at the University of Southampton is an important reflection of who I am"; $\alpha=.77 ; M=4.09, S D=$ 1.25); Private CSE (e.g., "Overall, I often feel that the social group of students at the University of Southampton is not worthwhile" [reverse coded]; $\alpha=.69 ; M=5.75, S D=0.81$ ); Public CSE (e.g., "In general, others respect the social group of students at the University of Southampton"; $\alpha=.63 ; M=5.37, S D=0.82)$; and Membership (e.g., "I am a worthy member of the University of Southampton community"; $\alpha=.62 ; M=5.20, S D=0.89$ ).

Finally, in a separate and ostensibly unrelated part of the study, we assessed participants' intentions to support the ingroup. They read about a publicity campaign that the university was planning to implement, where current students would be requested to contact former students and invite them to join an alumni society. They were then asked, "If approached to participate in the publicity campaign, approximately how many hours would you be willing to invest?" Participants indicated how many hours $(0-10)$ they would be willing to volunteer $(M=1.46 \mathrm{hr}, S D=1.90)$.

\section{Results}

We present means and standard deviations as a function of the event reflection and context manipulations in Table 2. Participant gender did not qualify any of the results reported in this section and was therefore omitted from the final analyses. Degrees of freedom vary due to missing values.

State nostalgia. A 2 (event reflection) $\times 2$ (context) ANOVA on state nostalgia produced a significant event-reflection main effect, $F(1,167)=12.16, p<.001, \eta^{2}=.07$. Across context, state nostalgia was higher when participants recalled a nostalgic (compared with ordinary) event. There was also a significant main effect of context, $F(1,167)=4.96, p=.027, \eta^{2}=.03$, indicating that state nostalgia was higher when participants recalled events that occurred in a personal (compared with collective) context. The Event Reflection $\times$ Context interaction was not significant, $F(1$, $167)=1.72, p=.192, \eta^{2}=.01$.

CSES total score. A 2 (event reflection) $\times 2$ (context) ANOVA on the CSES total score yielded a significant context main effect, $F(1,167)=6.79, p=.010, \eta^{2}=.04$, that was qualified by an Event Reflection $\times$ Context interaction, $F(1$, $167)=4.91, p=.028, \eta^{2}=.03$. Simple-effects tests showed that collective (compared with personal) nostalgia significantly increased CSE, $F(1,167)=10.93, p=.001, \eta^{2}=.06$. Recalling an ordinary collective (compared with personal) experience, however, did not increase CSE, $F(1,167)=0.08, p=.777, \eta^{2}=.001$.

CSES facets. A 2 (event reflection) $\times 2$ (context) ANOVA on Identity resulted in a significant Event Reflection $\times$ Context interaction only, $F(1,167)=9.50, p=.002, \eta^{2}=.05$. Simpleeffects tests showed that collective (compared with personal) nostalgia significantly increased the importance of ingroup membership to participants' self-concept, $F(1,167)=10.36, p=.002$, $\eta^{2}=.06$. Recalling an ordinary collective (compared with per-

Table 2

Means (Standard Deviations) as a Function of Event Reflection (Nostalgia vs. Ordinary) and Context (Collective vs. Personal) in Study 2

\begin{tabular}{|c|c|c|c|c|}
\hline \multirow{2}{*}{$\begin{array}{l}\text { Dependent } \\
\text { variable }\end{array}$} & \multicolumn{2}{|c|}{ Nostalgic event } & \multicolumn{2}{|c|}{ Ordinary event } \\
\hline & Collective & Personal & Collective & Personal \\
\hline Context check ${ }^{\mathrm{a}}$ & $4.61(1.15)$ & $4.82(1.19)$ & $4.37(1.38)$ & $5.01(1.04)$ \\
\hline State nostalgia & $4.33(1.62)$ & $5.17(1.24)$ & $3.81(1.64)$ & $4.03(1.61)$ \\
\hline CSES total score & $5.36(0.67)$ & $4.87(0.67)$ & $5.11(0.72)$ & $5.07(0.57)$ \\
\hline Identity & $4.51(1.06)$ & $3.63(1.33)$ & $3.96(1.50)$ & $4.23(0.94)$ \\
\hline Private CSE & $6.05(0.74)$ & $5.52(0.90)$ & $5.70(0.87)$ & $5.72(0.65)$ \\
\hline Public CSE & $5.54(0.75)$ & $5.31(0.87)$ & $5.46(0.84)$ & $5.20(0.80)$ \\
\hline Membership & $5.32(0.98)$ & $5.02(1.02)$ & $5.33(0.80)$ & $5.13(0.76)$ \\
\hline Hours volunteered & $2.27(2.63)$ & $0.97(1.27)$ & $1.37(1.51)$ & $1.25(1.73)$ \\
\hline \multicolumn{5}{|l|}{ LIWC } \\
\hline Word count & 83.54 (43.99) & $85.74(66.83)$ & 84.07 (66.17) & $78.54(60.24)$ \\
\hline LIWC-NA & $0.83(1.37)$ & $1.03(1.74)$ & $1.28(1.60)$ & $1.23(1.29)$ \\
\hline LIWC-PA & $5.77(2.91)$ & $5.81(4.19)$ & $4.58(4.35)$ & $4.32(3.99)$ \\
\hline
\end{tabular}

Note. $\quad$ CSES $=$ Collective Self-Esteem Scale; $\mathrm{CSE}=$ collective self-esteem; LIWC $=$ Linguistic Inquiry and Word Count; NA = negative affect. PA = positive affect.

${ }^{a}$ For context check, lower scores indicate greater salience of group membership. 
sonal) experience, however, did not, $F(1,167)=1.15, p=.285$, $\eta^{2}=.01$.

An ANOVA on Private CSE resulted in a significant context main effect, $F(1,167)=4.39, p=.038, \eta^{2}=.02$, that was qualified by an Event Reflection $\times$ Context interaction, $F(1$, $167)=5.13, p=.025, \eta^{2}=.03$. Tests of simple effects revealed that collective (compared with personal) nostalgia significantly strengthened participants' own judgments that the ingroup is a worthwhile entity, $F(1,167)=8.95, p=.003, \eta^{2}=.05$. Recalling an ordinary collective (compared with personal) experience, however, did not, $F(1,167)=0.02, p=.902, \eta^{2}=.0001$.

ANOVAs on Public CSE and Membership resulted in marginal context main effects. Ratings of Public CSE, $F(1,167)=3.87$, $p=.051, \eta^{2}=.02$, and Membership, $F(1,167)=3.35, p=.069$, $\eta^{2}=.02$, tended to be higher when participants recalled collective (compared with personal) experiences. No other effects were significant or marginal.

In summary, whereas collective (compared with personal) nostalgia significantly increased the importance of ingroup membership to participants' self-concept (Identity) and participants' own view that the group is a worthwhile entity (Private CSE), reflecting on an ordinary collective (compared with personal) experience did not. Reflecting on collective (compared with personal) experiences marginally increased perceived positive regard for the ingroup by outsiders (Public CSE) and the extent to which participants felt like worthy group members (Membership), irrespective of event type (nostalgia vs. ordinary). ${ }^{2}$

Support intentions. A 2 (event reflection) $\times 2$ (context) ANOVA on number of hours that participants were prepared to volunteer yielded a significant context main effect, $F(1,167)=$ $6.20, p=.014, \eta^{2}=.03$, that was qualified by an Event Reflection $\times$ Context interaction, $F(1,167)=4.24, p=.041, \eta^{2}=.02$. Simple-effects tests showed that collective (compared with personal) nostalgia significantly strengthened support intentions, $F(1$, $167)=9.73, p=.002, \eta^{2}=.06$. Recalling an ordinary collective (compared with personal) experience, however, did not influence support intentions, $F(1,167)=0.10, p=.752, \eta^{2}=.001$. Thus, whereas collective (compared with personal) nostalgia strengthened support intentions, reflecting on an ordinary collective (compared with personal) experience did not. ${ }^{3}$

LIWC emotion categories. We submitted the participantgenerated autobiographical narratives to LIWC. The average length of the narratives was 82.75 words $(S D=59.50)$. A 2 (event reflection) $\times 2$ (context) ANOVA on word count yielded no significant or marginal effects, $F \mathrm{~s}(1,166) \leq 0.18, p \mathrm{~s} \geq .676$. Next, we performed ANOVAs on LIWC-NA and LIWC-PA. For LIWC-NA, there were no significant or marginal effects, $F_{\mathrm{S}}(1$, $166) \leq 2.01, p s \geq .158$. For LIWC-PA, there was a significant main effect for event reflection only, $F(1,166)=4.97, p=.027$, $\eta^{2}=.03$. Across context, nostalgic narratives contained a greater proportion of positive emotion words than did ordinary narratives. Finally, it is noteworthy that written expressions of PA were much more frequent than expressions of NA in all four conditions, $F_{\mathrm{S}}(1$, $166) \geq 23.51, p s<.001$. Thus, by instructing participants to recall an ordinary collective or personal event, we did not inadvertently prompt them to think of experiences that were negative.

Mediational analyses. We present zero-order correlations among the CSES (total score and facets), LIWC-NA and LIWC-PA categories, and support intentions in Table 3. Correla- tions among the CSE facets, including the relatively low (but significant) correlation between Identity and Public CSE, closely resemble those reported by Luhtanen and Crocker (1992).

CSES total score. Collective (compared with personal) nostalgia increased CSE (as assessed by the CSES total score), but reflecting on an ordinary collective (compared with personal) experience did not. Furthermore, CSE was significantly correlated with support intentions. These results set the stage for testing whether CSE mediated the specific effect of collective (compared with personal) nostalgia on support intentions. To examine mediation, we tested a model that has been discussed by Edwards and Lambert (2007, p. 4) as "direct effect and first stage moderation model" and by Hayes (2013) as Model 8. We depict this model in Figure 1 (top panel). Specifically, we used the PROCESS macro (Hayes, 2013, Model 8) in SAS 9.3 to calculate bootstrap confidence intervals (CIs) for the indirect effect (denoted as $a b$ ) of context (collective vs. personal) on support intentions via CSE, conditional upon event reflection (nostalgia vs. ordinary). For participants who recalled an ordinary event, this indirect effect was not significant, $a b=.011,95 \%$ CI $[-.080, .010]$. For participants who recalled a nostalgic event, this indirect effect was significant, $a b=.139,95 \%$ CI $[.043, .311]$. In the nostalgia condition, the direct effect of context (collective vs. personal) on support intentions was also significant, $B=0.508, S E=0.210, t(166)=2.42$, $p=.017, \eta^{2}=.03$. Note that, by using the term indirect effect, we have adopted the parlance of intervening variable models and did not mean to claim support for causality. Further note that, following Hayes (2013), we did not adopt the traditional distinction between partial and full mediation. In all, these results are consistent with a mediational sequence whereby collective (compared with personal) nostalgia increases CSE, which, in turn, increases support intentions.

CSES facets. Collective (compared with personal) nostalgia increased the importance of ingroup membership to participants' self-concept (as assessed by Identity) and participants' own regard for the ingroup (as assessed by Private CSE), but reflecting on an ordinary collective (compared with personal) experience did not. Identity (but not Private CSE) scores were also significantly correlated with support intentions, suggesting a possible mediational role. We used the PROCESS macro (Hayes, 2013, Model 8) to test

\footnotetext{
${ }^{2}$ From a different vantage point, the Event Reflection $\times$ Context interaction effect on the CSES total score showed that in the collective context, reflecting on a nostalgic (compared with ordinary) event marginally increased CSE, $F(1,167)=2.84, p=.094, \eta^{2}=.02$. In the personal context, however, reflecting on a nostalgic (compared with ordinary) event did not significantly influence CSE, $F(1,167)=2.10, p=.149, \eta^{2}=.01$ For the Identity facet, results indicated that reflecting on a nostalgic (compared with ordinary) event increased the importance of ingroup membership in the collective context, $F(1,167)=4.22, p=.042, \eta^{2}=.02$, bu reduced it in the personal context, $F(1,167)=5.32, p=.022, \eta^{2}=.03$. For the Private CSE facet, results showed that recalling a nostalgic (compared with ordinary) event strengthened perceptions of the ingroup as a worthwhile entity in the collective context, $F(1,167)=3.99, p=.047$, $\eta^{2}=.02$, but not in the personal context, $F(1,167)=1.44, p=.232, \eta^{2}=$ .01

${ }^{3}$ From a different vantage point, the Event Reflection $\times$ Context interaction effect showed that reflecting on a nostalgic (compared with ordinary) event increased support intentions in the collective context, $F(1$, 167) $=4.90, p=.028, \eta^{2}=.03$, but not in the personal context, $F(1$, 167) $=0.48, p=.489, \eta^{2}=.002$.
} 
Table 3

Zero-Order Correlations Among CSES (Total Score and Facets), LIWC Negative and Positive Emotion Categories, and Support Intentions (Hours Volunteered) in Study 2

\begin{tabular}{|c|c|c|c|c|c|c|c|c|}
\hline Variable & 1 & 2 & 3 & 4 & 5 & 6 & 7 & 8 \\
\hline 1. CSES total score & - & & & & & & & \\
\hline 2. Identity & $.69^{\text {**** }}$ & - & & & & & & \\
\hline 3. Private CSE & $.81^{\text {****** }}$ & $.36^{* * * *}$ & - & & & & & \\
\hline 4. Public CSE & $.67^{* * * * *}$ & $.16^{*}$ & $.56^{* * * *}$ & - & & & & \\
\hline 5. Membership & $.70^{\text {***** }}$ & $.21^{\text {** }}$ & $.53^{* * * *}$ & $.38^{* * * * *}$ & - & & & \\
\hline 6. LIWC-NA & .04 & .01 & .08 & .05 & .00 & - & & \\
\hline 7. LIWC-PA & .07 & .04 & $.16^{*}$ & .11 & -.09 & $-.19^{*}$ & - & \\
\hline 8. Hours volunteered & $.25^{\text {****** }}$ & $.26^{* * * *}$ & .11 & $.21^{\text {*** }}$ & .10 & .09 & -.00 & - \\
\hline
\end{tabular}

Note. $\quad N=171$. For Linguistic Inquiry and Word Count-negative affect (LIWC-NA) and LIWC-positive affect (LIWC-PA), $N=170$ due to a missing narrative. CSES $=$ Collective Self-Esteem Scale.

${ }^{*} p<.05$. ** $p<.01$. $^{* * * *} p<.001$.

the indirect effect of recalling collective (compared with personal) experiences on support intentions via Identity and Private CSE, conditional upon event reflection (nostalgia vs. ordinary). We conducted both simple mediational analyses (i.e., entering the mediators in separate analyses) and a parallel mediational analysis (i.e., entering the mediators simultaneously). We present the results of these analyses in Table 4. For participants who recalled a nostalgic event, there was a significant indirect effect of context (collective vs. personal) on support intentions via Identity (but not via Private CSE). For participants who recalled an ordinary event, this indirect effect was not significant. The simple and parallel mediational analyses produced similar results. In summary, results are consistent with a mediational sequence whereby collective (compared with personal) nostalgia increases the importance of ingroup membership to participants' self-concept, which, in turn, increases support intentions.
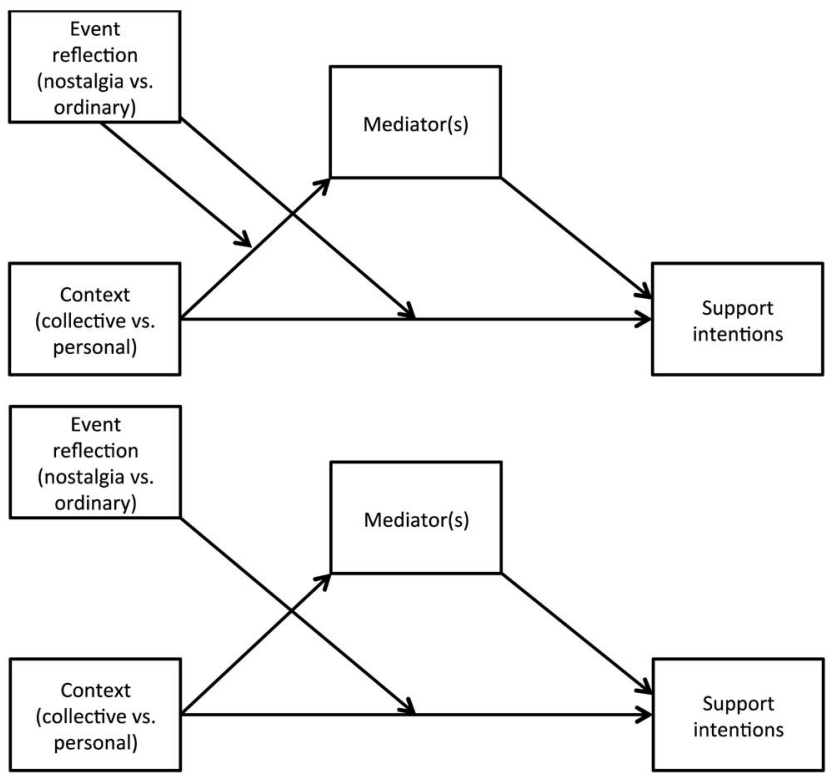

Figure 1. Schematic representation of the direct effect and first stage moderation model (top panel) and the direct effect moderation model (bottom panel) tested in Study 2.
There were no significant Event Reflection $\times$ Context interaction effects on the Public CSE and Membership facets. Instead, we obtained marginal context main effects, such that bringing to mind collective (compared with personal) experiences tended to increase Public CSE and Membership ratings, irrespective of event type (nostalgia vs. ordinary). Public CSE (but not Membership) ratings were also significantly correlated with support intentions, suggesting a possible mediational role. Accordingly, we tested a model that has been discussed by Edwards and Lambert (2007, p. 9) as "direct effect moderation model" and by Hayes (2013) as Model 5. We depict this model in Figure 1 (bottom panel). We used the PROCESS macro (Hayes, 2013, Model 5) to test the indirect effect of context (collective vs. personal) on support intentions via Public CSE and Membership. Table 4 shows that there was a significant indirect effect of context (collective vs. personal) on support intentions via Public CSE (but not via Membership) in both the simple and parallel mediational analyses. To summarize, these results are consistent with a mediational sequence whereby recalling collective (compared with personal) experiences increases perceived positive regard for the ingroup by outsiders, which, in turn, increases support intentions. ${ }^{4}$

LIWC emotion categories. Nostalgic narratives contained a greater proportion of positive emotion words than did ordinary narratives. Could this account for our findings? The low correlations of LIWC-PA with the CSES facets and support intentions render this possibility unlikely. Indeed, when we controlled for LIWC-PA by including it as a covariate in the mediational analyses, the results were essentially unchanged. To conclude, as in Study 1, we found no support for a role of PA.

\section{Discussion}

Study 2 provided additional evidence for the discriminant validity of the collective nostalgia construct by further distinguishing it from remembering of collective experiences per se. Whereas collective (compared with personal) nostalgia increased support

\footnotetext{
${ }^{4}$ Supplementary analyses revealed that the mediational role of Identity was also supported when Public CSE was included as a covariate and vice versa. All mediational analyses found that, in the nostalgia condition, the direct effect of context (collective vs. personal) on support intentions was significant
} 
Table 4

Indirect Effects [95\% Bootstrapped Confidence Interval] of Context (Collective vs. Personal) on Support Intentions Via CSES Facets in Study 2

\begin{tabular}{|c|c|c|}
\hline $\begin{array}{c}\text { Mediator (CSES facet)/Level of moderator } \\
\text { (event reflection) }\end{array}$ & $\begin{array}{l}\text { Simple mediational } \\
\text { analysis }\end{array}$ & $\begin{array}{l}\text { Parallel mediational } \\
\text { analysis }\end{array}$ \\
\hline \multicolumn{3}{|l|}{ First stage and direct effect moderation-Model 8} \\
\hline \multicolumn{3}{|l|}{ Identity } \\
\hline Nostalgia & $.145^{*}[.035, .325]$ & $.149^{*}[.032, .349]$ \\
\hline Ordinary & $-.046[-.201, .025]$ & $-.047[-.192, .030]$ \\
\hline \multicolumn{3}{|l|}{ Private collective self-esteem } \\
\hline Nostalgia & $.035[-.050, .150]$ & $-.010[-.129, .066]$ \\
\hline Ordinary & $-.001[-.047, .023]$ & $.000[-.025, .037]$ \\
\hline \multicolumn{3}{|l|}{ Direct effect moderation-Model 5} \\
\hline Public collective self-esteem: No moderated mediation & $.052 *[.004, .137]$ & $.051^{*}[.004, .156]$ \\
\hline Membership: No moderated mediation & $.021[-.012, .106]$ & $.003[-.041, .068]$ \\
\hline
\end{tabular}

intentions, remembering regular, ordinary collective (compared with personal) experiences did not. This beneficial effect of collective nostalgia was mediated by CSE. Although the limitations of mediational analyses are now well documented (Bullock, Green, \& $\mathrm{Ha}, 2010)$, we nonetheless consider them informative because they placed our predictions concerning the role of CSE at risk (Fiedler, Schott, \& Meiser, 2011). These findings add to a growing body of evidence that CSE predicts tangible expressions of group and organizational commitment (Blader \& Tyler, 2009; Ellemers et al., 1999; Fuller et al., 2006; de Moura et al., 2009). We examined unobtrusively the role of PA by controlling for the relative frequency of discrete positive and negative emotion terms in participants' narratives. These analyses again failed to support the idea that the beneficial effects of collective nostalgia are due merely to the PA it entails.

More fine-grained analyses, focusing on the CSES facets, shed light on the specific mechanisms linking collective nostalgia with support intentions. Results revealed that collective (compared with personal) nostalgia increased the importance of ingroup membership to participants' self-concept, which, in turn, predicted support intentions. This effect of collective (compared with personal nostalgia) on the importance of ingroup membership is noteworthy, because the significance of a naturally occurring ingroup identity to the self-concept is solidified by prior experience, and there is scant evidence that it can be manipulated within the brief timespan of a typical experiment (Ellemers et al., 2002; McCoy \& Major, 2003; Ramos et al., 2014). The present findings, then, open a path to experimental research on the pivotal role of ingroup identity in group processes and intergroup relations. To illustrate this potential, we note that recent studies found that Greek participants who recalled a nostalgic (compared with ordinary) experience they shared with other Greeks showed an increase in ethnocentric product preferences. That is, collective nostalgia increased their ratings of domestic products (e.g., songs, television shows) and reduced ratings of similar foreign products (Dimitriadou, Maciejowski, Wildschut, \& Sedikides, 2014).

Study 2 also demonstrated that collective (compared with personal) nostalgia strengthened participants' own judgments that the ingroup is a worthwhile entity (as assessed by Private CSE), whereas remembering ordinary collective (compared with per- sonal) experiences did not. However, participants' regard for the ingroup did not predict support intentions, and accordingly, it did not play a mediational role. The finding that the importance of ingroup membership to participants' self-concept (Identity) but not participants' regard for the ingroup (Private CSE), mediated the effect of collective (compared with personal) nostalgia on support intentions has both theoretical and practical significance. From a theoretical standpoint, the finding lends support to the notion that overlap between cognitive representations of self and ingroup facilitates tangible sacrifices on behalf of the ingroup, because it aligns self-interest with group interest (Abrams, 2013; Tropp \& Wright, 2001). From a practical point of view, the present findings indicate that collective nostalgia can be implemented effectively to increase constructive citizenship behavior and reduce turnover in organizational contexts by targeting organizational identity (as opposed to positive regard for the ingroup). For instance, employees could be encouraged to recall nostalgic experiences that epitomize their belongingness to the organization (rather than its positive qualities).

Study 2 showed that recalling collective (compared with personal) experiences per se (irrespective of whether the experience was nostalgic or ordinary) indirectly increased support intentions via participants' perceptions of outsiders' positive regard for the ingroup (as assessed by Public CSE). Although this finding does not explain why collective (compared with personal) nostalgia increased support intentions more so than did recalling an ordinary collective (compared with personal) experience, it is nonetheless important, if only because collective nostalgia partakes in this more general beneficial indirect effect of recalling collective experiences. As far as we are aware, this is the first evidence that recollection of collective (compared with personal) experiences can increase meta-perceptions of outsiders' positive regard for the ingroup. The link between such positive meta-perceptions and support intentions is consistent with Tyler and Blader's (2003) group engagement model, which posits that the perceived status and prestige accorded to an organization by outsiders promotes psychological engagement among members, thereby enhancing favorable attitudes toward the organization, adoption of organizational values, and manifestation of citizenship behavior to support the organization (Blader \& Tyler, 2009; Fuller et al., 2006). 
Finally, from a different vantage point, Study 2 showed that reflecting on a nostalgic (compared with ordinary) event increased CSE (in particular, the Identity and Private CSE facets) and support intentions in the collective context but not in the personal context (see footnotes 2 and 3). Thus, the collective-nostalgia condition differed significantly from the personal-nostalgia as well as the collective-ordinary condition. Inspection of Table 2 indicates that the collective-ordinary condition provides a more stringent benchmark than the personal-nostalgia condition for assessing the effects of collective nostalgia. For this reason, we focused on a comparison between collective nostalgia and recall of ordinary collective experiences in Study 3.

\section{Study 3}

The preceding studies demonstrated that collective (compared with personal) nostalgia improves ingroup evaluation, strengthens global inclinations to approach (and not avoid) ingroup members, and promotes specific behavioral intentions to support the ingroup. These ingroup-benefiting effects of collective nostalgia were independent of PA and went above and beyond the effect of recalling positive or ordinary collective events (Studies 1 and 2). On this basis, we concluded that collective nostalgia met two criteria for qualifying as a grouplevel emotion: it (a) can be differentiated from analogous individual-level emotions and (b) motivates and regulates attitudes and behavior in relation to social groups. In Study 3, we extended this line of research in three ways. First, we examined whether collective nostalgia meets a third criterion for qualifying as a group-level emotion. Smith et al. (2007) proposed that the experience and expression of group-level emotions are functions of the individual's level of social identification with the group. Accordingly, we assessed social identification and examined whether the effect of collective nostalgia (compared with recalling an ordinary collective event) on group processes is stronger when social identification is high (compared with low). Second, we moved beyond our prior focus on global action tendencies (Study 1) and specific behavioral intentions (Study 2) to assess concrete behavior. By so doing, we took another important step toward the ultimate objective of mapping the implications of collective nostalgia for group-related behavior. Specifically, we used the third-party punishment game (Fehr \& Fischbacher, 2004), in which an allocator has transferred a small number of valuable tokens to a member of the participants' ingroup and kept a much larger number of tokens to herself or himself, thereby violating fairness norms. Participants could then sacrifice their own valuable tokens to punish the allocator's transgression against the ingroup member, thereby incurring a tangible financial cost on behalf of the ingroup. Third, whereas in the preceding studies we instructed participants to consider a university ingroup, in the present study, we involved a national (i.e., Irish) ingroup to establish the generalizability of the role of collective nostalgia. We hypothesized that collective nostalgia (compared with recalling an ordinary collective event) would increase more strongly the number of valuable tokens participants would sacrifice to punish a transgression against an ingroup member when social identification with the ingroup is high (compared with low).

\section{Method}

Participants and design. We randomly assigned 49 University of Limerick undergraduates ( 27 females, 22 males; $M_{\text {age }}=$ 19.84 years, $S D_{\text {age }}=2.26$ ) to the collective-nostalgia or collective-ordinary condition. Participants (all Irish) were remunerated with 4 euros.

Procedure and materials. Participants learned that the research session consisted of three separate studies. First, they completed a "personality study," which provided the context for assessing social identification. As part of this study, participants filled out the social identification scale by Tarrant, North, and Hargreaves (2004). This scale comprised 13 items that measured identification with the target group - the Irish (e.g., "I identify with this group," and "I think this group is important"; $1=$ strongly disagree, 10 = strongly agree $)$. We averaged the items to form a social identification index $(\alpha=.84 ; M=3.16, S D=1.53)$. We included filler questionnaires before and after the social identification measure to obscure the true purpose of this "personality study."

Then, we informed participants that they would take part in a "computer game study." Here, we introduced the third-party punishment game that provided the context for assessing the cost participants were willing to incur to punish a transgression against an ingroup member. Participants learned that the game involved a set of three players: an allocator, a receiver, and an observer. The instructions stated that the allocator, receiver, and observer would be endowed with 40, 0, and 20 tokens, respectively. We informed participants that we would recruit a total of 50 three-player sets over the entire course of the study and that we would randomly select one of these sets to receive prize money. The tokens represented the amount of money (in euros) participants would be awarded in the event that their set was selected to receive the prize money. We rendered salient the ingroup membership of the receiver by means of a procedure developed by van Tilburg and Igou (2011), which capitalized on the fact that a particular spelling of a person's first name can convey information about their nationality. In the present study, we told Irish participants that the receiver was named Eoin, which is the typical Irish spelling of the more common name Owen.

We informed participants that role assignment was random, but, in actuality, all of them were assigned to the role of observer. Participants read that at the start of the game, the allocator could transfer some or all of the 40 tokens to the receiver. Note that if the allocator transferred 20 tokens to the receiver, all three players in the set could potentially exit the game with an equal number of tokens (20 tokens each). Next, the observer would be allowed to punish the allocator by sacrificing tokens. Specifically, the allocator would lose two tokens for each token the observer sacrificed to punish the allocator. We made clear that this punishment would affect participants' own balance and that of the allocator, but not that of the receiver. Participants then read a detailed example to ensure their understanding of the rules.

Next, we told participants that the other two players in the three-player set would be selected from two other research locations. To bolster the credibility of these instructions, we asked participants to enter their first name and a number representing their research location, which they could request from the experimenter. After entering this information, participants were shown a 
message on the computer screen that indicated that the computer was connecting with the other research locations to search for available players. After $1 \mathrm{~min}$, a message indicated that two players had been identified and that the game would start once these other players had finished reading the instructions. We instructed participants that, in the interim, they would complete a brief, unrelated study. This "memory study" provided the context for the collective-nostalgia induction. Participants recalled either a nostalgic event that they had experienced together with other Irish people (collective-nostalgia condition) or an ordinary event that they had experienced together with other Irish people (collectiveordinary condition). Following this, they completed the two-item measure of state nostalgia (see preceding studies; $1=$ not at all, $6=$ very much; $\alpha=.91 ; M=3.79, S D=1.30$ ).

A message then indicated that the computer was connecting to the other players and that the participant had been assigned to the role of observer. In that role, participants were endowed with 20 tokens. We reminded participants that the person in the role of allocator had an endowment of 40 tokens and the person in the role of receiver had 0 tokens. A message indicated that the allocator was named Owen, and the receiver was named Eoin, thereby rendering salient the ingroup membership of the receiver (van Tilburg \& Igou, 2011). At this point, the game commenced and participants read that the allocator, Owen, had transferred only five tokens of his endowment to the receiver, Eoin, and kept 35 tokens to himself. Participants then indicated how many tokens they wanted to spend on punishing Owen's unfair transgression against Eoin $(M=6.49, S D=4.94)$.

\section{Results}

State nostalgia. We entered the measure of state nostalgia as dependent variable in a moderated regression analysis. Event reflection (contrast coded: collective nostalgia $=1$, collective ordinary $=-1$ ), social identification (mean centered), and the Event Reflection $\times$ Social Identification interaction were the independent variables. As intended, the regression analysis yielded a significant main effect of event reflection only, $\beta=0.42, t(45)=$ 2.87, $p=.006, \eta^{2}=.15$. Participants in the collective-nostalgia condition reported a higher level of state nostalgia $(M=4.24$, $S D=1.19)$ than did participants in the collective-ordinary condition $(M=3.31, S D=1.25)$. Neither the main effect of social identification, $\beta=-0.17, t(45)=-1.10, p=.277, \eta^{2}=.02$, nor the Event Reflection $\times$ Social Identification interaction, $\beta=0.15$, $t(45)=1.05, p=.301, \eta^{2}=.02$, were significant. Thus, the collective nostalgia induction was successful, irrespective of social identification.

Punishment of transgression against an ingroup member. We entered the number of tokens participants sacrificed to punish the transgression against the ingroup member (i.e., Eoin) as dependent variable in a moderated regression analysis. Results indicated a significant Event Reflection $\times$ Social Identification interaction only, $\beta=0.32, t(45)=2.20, p=.033, \eta^{2}=.09$. The main effects of event reflection, $\beta=.12, t(45)=0.86, p=.397, \eta^{2}=$ .01 , and social identification, $\beta=.12, t(45)=0.81, p=.424$, $\eta^{2}=.01$, were not significant. We depict the Event Reflection $\times$ Social Identification interaction in Figure 2. Simple-effects tests revealed that when social identification was high (1 $S D$ above the mean), participants in the collective-nostalgia condition sacrificed

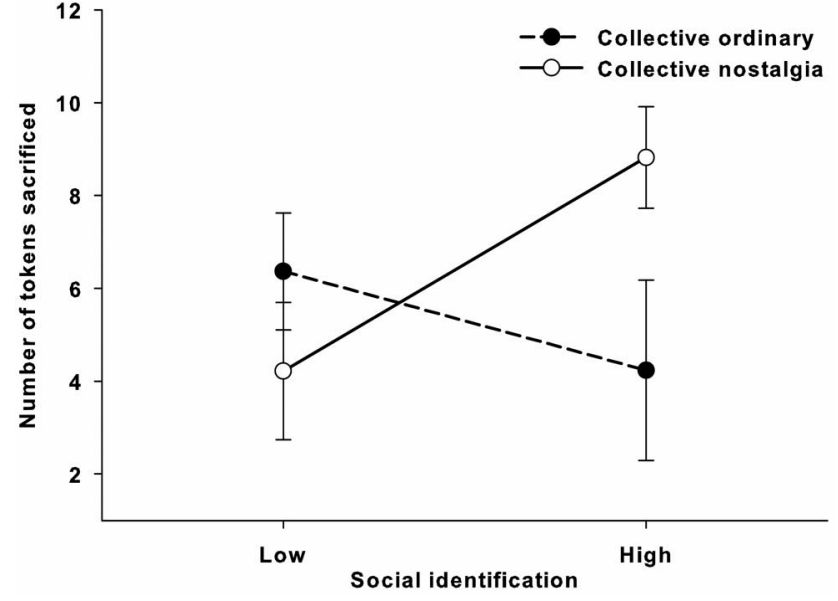

Figure 2. Number of tokens sacrificed to punish transgressions against an ingroup member as a function of collective nostalgia (compared with recalling ordinary collective events) and social identification in Study 3. Plotted values are predicted means conditioned at 1 standard deviation $(S D)$ below (low social identification) and $1 S D$ above (high social identification) the mean of social identification. Error bars represent standard errors.

more tokens to punish the transgression against Eoin than did participants in the collective-ordinary condition, $\beta=0.47, t(45)=$ 2.06, $p=.046, \eta^{2}=.08$. When social identification was low (1 $S D$ below the mean), however, the difference between the collective-nostalgia and collective-ordinary conditions was not significant, $\beta=-0.22, t(45)=-1.11, p=.275, \eta^{2}=.02$. The Johnson-Neyman region of significance (Hayes, 2013) starts at a raw score of 4.55 . This denotes that for participants with a raw score greater than 4.55 on social identification, recalling nostalgic (compared with ordinary) events shared with other ingroup members is predicted to significantly $(p<.05)$ increase punishment.

From a different vantage point, the significant interaction effect indicated that for participants in the collective-nostalgia condition, social identification was associated with stronger punishment of a transgression against an ingroup member, $\beta=0.47, t(45)=2.66$, $p=.011, \eta^{2}=.13$. For participants in the ordinary-collective condition, however, the association between social identification and punishment was not significant, $\beta=-0.22, t(45)=-0.84$, $p=.403, \eta^{2}=.01$.

\section{Discussion}

Study 3 demonstrated that collective nostalgia meets a third criterion for qualifying as a group-level emotion. Results supported the idea that collective nostalgia has more far-reaching implications for group processes when social identification with the ingroup is high (compared with low). This evidence was obtained within the context of a task in which participants made tangible financial sacrifices to punish someone (Owen) who treated an ingroup member (Eoin) unfairly. For Irish participants who were highly identified with their national ingroup, collective nostalgia (compared with recalling an ordinary collective event) increased the financial costs incurred to punish a transgression perpetrated against an ingroup member. When identification with 
the national ingroup was low, the effect of collective nostalgia was not significant.

In Study 2, ingroup support was significantly stronger in the collective-nostalgia than in the collective-ordinary condition. In Study 3, we conceptually replicated this effect only when social identification was high. Across levels of social identification, the difference between the collective-nostalgia and collective-ordinary conditions (i.e., the event reflection main effect) was not significant. Although we did not expect this null finding, it is nonetheless consistent with the notion that it is easier to characterize grouplevel emotions in terms of participants' readiness or inclination to engage in certain types of behavior than in terms of actual behavioral manifestations (Mackie et al., 2000). As previously mentioned, one reason for this is that actual behaviors are more constrained by situational factors than are action tendencies or intentions. Another key reason is that action tendencies and intentions can be channeled into a variety of behaviors, and it is difficult to predict precisely these different behavioral manifestations. In the present context, it is possible that some participants reached the conclusion that they could better support the ingroup as a whole by saving, rather than sacrificing, their valuable tokens because sacrificing tokens to punish the perpetrator (Owen) would not increase outcomes for the ingroup victim (Eoin) but would diminish their own outcomes. This illustrates the challenges of studying group-related behavior.

\section{Study 4}

Thus far, we have presented evidence that collective nostalgia meets three criteria for qualifying as a group-level emotion. Study 4 completed this line of research by examining the fourth, and final, criterion: group-level emotions are socially shared or converge within a group. Smith et al. (2007) envisioned that this convergence would go "beyond the idea that a small group of people who interact face to face might influence each other's moods or emotions" (p. 433). Rather, they proposed that the convergence of group-level emotions should also occur for larger and more abstract social categories, such as one's nationality. In the preceding studies, we operationalized collective nostalgia by instructing participants to recall a nostalgic memory of an experience they shared with other ingroup members. Whereas the explicit focus on shared experiences was sufficient to make participants think of themselves in terms of a particular group membership (see Study 2 manipulation check), we do not consider it necessary. IET (Mackie et al., 2000; Mackie \& Smith, 1998; Smith, 1993, 1999) proposes that when individuals think of themselves as part of a group, that group and events or objects related to it acquire emotional significance. Accordingly, in Study 4, we operationalized collective nostalgia by instructing members of the U.S. general public to think of themselves as a member of their national group and then indicate to what extent they generally experience nostalgia. By so doing, we were able to assess the convergence of collective nostalgia among members of a larger, abstract social category and move beyond the more specific focus on shared experiences in face-to-face groups.

We examined collective nostalgia in conjunction with other individual and group-level emotions. This allowed us to compare and contrast collective nostalgia to (a) individual nostalgia and (b) other group-level emotions. A supplementary objective of Study 4 therefore was to determine if participants identified collective nostalgia as a discrete emotion.

\section{Method}

Participants. We recruited 108 US participants (64 men, 43 women, 1 unreported; $M_{\text {age }}=35.28$ years, $\left.S D_{\text {age }}=11.57\right)$ via Amazon's Mechanical Turk (MTurk). All participants had a track record of $95 \%$ or better job acceptance rate and were paid $\$ 0.10$. We analyzed the data of participants who completed all emotion ratings, resulting in a final sample of 96 participants (56 men, 39 women, and one person whose gender was unreported; $M_{\text {age }}=$ 34.60 years, $S D_{\text {age }}=11.56$ ).

Procedure and materials. Participants completed materials online. We first instructed them to rate 13 emotions for the extent to which they felt each of these emotions as an individual $(1=n o t$ at all, 7 = very much). Instructions read: "When you think about yourself as an individual, to what extent do you feel each of the following emotions?" Twelve of the emotions were identical to those used by Smith et al. (2007): angry, satisfied, afraid, hopeful, proud, disgusted, uneasy, happy, grateful, guilty, respectful, and irritated. We added the 13th emotion: nostalgic. We then assessed group-level emotions by asking participants about their emotions as an American: "When you think about yourself as an American, to what extent do you feel each of the following emotions?" Participants then rated the same 13 emotions as before. Smith et al. (2007, Study 1) found that the order in which the individual and group-level emotions were assessed (individual emotions first vs. group-level emotions first) did not influence results, and we therefore used the fixed order described previously.

\section{Results}

Preliminary analyses showed that gender and age did not have substantive effects, with one exception (footnoted later). Neither gender nor age was significantly correlated with individual- or group-level nostalgia. All analyses therefore collapsed across gender and age.

Collective nostalgia is shared within groups. An intuitive measure of the extent to which a particular group-level emotion converges toward a group prototype or average is the standard deviation. If convergence is high, the standard deviation will be low. Table 5 shows that the standard deviation for group-level nostalgia was comparatively low $(S D=1.60)$. Only group-level fear ("afraid") and satisfaction ("satisfied") had numerically lower standard deviations. We formally compared the standard deviation of group-level nostalgia to those of the other 12 group-level emotions, using Pitman's (1939) test (Lee, 1992). The standard deviation of group-level nostalgia did not differ significantly from the other standard deviations ( $p$ s $>.077$ ). The standard deviation for individual nostalgia was comparatively high $(S D=1.74)$. Thus, there was no evidence to suggest that ratings of nostalgia generally manifest high convergence, regardless of whether participants think of themselves as a member of their group or as a unique individual. We regard this as prima facie evidence that collective nostalgia is shared within groups to the same extent as other group-level emotions.

We also considered all emotion ratings simultaneously and assessed the extent to which participants' group-level emotions 
Table 5

Descriptive Statistics and Zero-Order Correlations Among Measured Variables in Study 4

\begin{tabular}{|c|c|c|c|c|c|c|c|c|c|c|c|c|c|c|c|c|c|}
\hline \multirow[b]{2}{*}{ Emotion } & \multicolumn{2}{|c|}{ Individual } & \multicolumn{2}{|c|}{ Group-level } & \multicolumn{13}{|c|}{$r_{\text {emotion }}$} \\
\hline & $M$ & $S D$ & $M$ & $S D$ & 1 & 2 & 3 & 4 & 5 & 6 & 7 & 8 & 9 & 10 & 11 & 12 & 13 \\
\hline 1. Angry & 2.55 & 1.61 & 3.03 & 1.76 & .63 & .76 & .76 & .66 & .54 & .74 & -.01 & .08 & -.09 & .04 & .03 & .03 & .26 \\
\hline 2. Afraid & 2.30 & 1.44 & 2.53 & 1.56 & .70 & .69 & .68 & .73 & .60 & .67 & -.16 & .07 & -.20 & -.07 & -.05 & -.08 & .20 \\
\hline 3. Disgusted & 2.25 & 1.42 & 2.99 & 1.76 & .81 & .72 & .50 & .74 & .59 & .72 & -.14 & -.02 & -.19 & -.08 & -.08 & -.12 & .30 \\
\hline 4. Uneasy & 2.66 & 1.59 & 2.99 & 1.73 & .73 & .79 & .76 & .66 & .62 & .80 & -.20 & -.04 & -.21 & -.03 & -.02 & -.07 & .30 \\
\hline 5. Guilty & 2.02 & 1.28 & 2.30 & 1.60 & .39 & .49 & .45 & .49 & .55 & .53 & -.09 & -.11 & -.20 & -.12 & -.19 & -.08 & .17 \\
\hline 6. Irritated & 2.67 & 1.58 & 3.35 & 1.92 & .77 & .70 & .84 & .78 & .45 & .62 & -.17 & .01 & -.20 & -.07 & -.02 & -.09 & .32 \\
\hline 7. Satisfied & 4.30 & 1.59 & 4.10 & 1.55 & -.30 & -.23 & -.45 & -.33 & -.03 & -.35 & .62 & .82 & .79 & .72 & .65 & .79 & .24 \\
\hline 8. Proud & 4.26 & 1.69 & 4.32 & 1.80 & -.20 & -.22 & -.42 & -.29 & -.13 & -.28 & .77 & .53 & .69 & .64 & .66 & .67 & .34 \\
\hline 9. Happy & 4.67 & 1.59 & 4.41 & 1.71 & -.29 & -.25 & -.46 & -.36 & -.24 & -.37 & .79 & .87 & .66 & .81 & .75 & .77 & .21 \\
\hline 10. Grateful & 4.85 & 1.81 & 4.75 & 1.83 & -.13 & -.15 & -.31 & -.23 & -.13 & -.26 & .69 & .78 & .85 & .68 & .83 & .81 & .37 \\
\hline 11. Respectful & 4.95 & 1.68 & 4.71 & 1.76 & -.20 & -.19 & -.40 & -.27 & -.20 & -.29 & .72 & .84 & .88 & .86 & .71 & .79 & .49 \\
\hline 12. Hopeful & 4.92 & 1.84 & 4.66 & 1.79 & -.19 & -.13 & -.37 & -.21 & -.04 & -.28 & .64 & .71 & .77 & .76 & .80 & .71 & .32 \\
\hline 13. Nostalgic & 4.27 & 1.74 & 4.00 & 1.60 & .04 & .02 & -.11 & -.03 & -.11 & -.07 & .35 & .49 & .56 & .53 & .61 & .45 & .49 \\
\hline
\end{tabular}

Note. Correlations among group-level emotions are below the diagonal and correlations among individual emotions are above the diagonal. Correlations between corresponding group-level and individual emotions are on the diagonal (in boldface). Degrees of freedom for correlations $\left(r_{\text {emotion}}\right): 96-2=94$. Correlations equal to or greater than $|.20|$ are statistically significant $(p<.05)$.

(including nostalgia) aligned with the average or prototypical group-level emotion profile. If group-level emotions are shared within a group, the average group-level emotion profile should predict participants' group-level emotions and do so above and beyond their individual emotions. We used hierarchical linear modeling (HLM) to test this prediction. The multilevel data set comprised 13 emotions (Level 1) nested within participant (Level 2). We modeled each participant's rating of a particular grouplevel emotion as a function of two Level 1 predictors: (a) the average rating across participants of that particular group-level emotion and (b) the participant's rating of the corresponding individual-level emotion. We implemented this HLM analysis in SAS PROC MIXED (for details of this analytic approach, see Smith et al., 2007). ${ }^{5}$ Participants' ratings of individual emotions significantly predicted their ratings of the corresponding grouplevel emotions, $B=0.45, S E=0.05, t(1150)=9.53, p<.001$. More important, the prototypical American emotion profile also significantly predicted participants' ratings of group-level emotions, above and beyond ratings of individual emotions, $B=0.41$, $S E=0.10, t(1150)=4.05, p<.001$. In all, these findings further support the idea that group-level emotions-including nostalgiaare socially shared. To illustrate this, imagine an individual who scores 2 on individual nostalgia, whereas the prototypical grouplevel score is 4 . This individual would have a predicted score of 3.11 for group-level nostalgia, thus covering over half the distance between his or her individual-level rating and the group-level prototype. (We calculated this predicted mean from the full centered equation, including the intercept).

Group-level nostalgia is distinct from individual nostalgia. Whereas the key objective of this study was to examine Criterion 4 , it also presented an opportunity to garner additional evidence for the distinction between collective and personal nostalgia (Criterion 1). The above-described HLM analysis showed a robust positive association between individual- and group-level emotions, which raises a legitimate concern that the latter are merely reflections of their individual counterparts. To investigate this formally, we converted the multiple-record multilevel data set into a multiplevariable data set, in which ratings of individual and group-level emotions were treated as repeated measures (13 individual and 13 group-level emotion ratings per participant). We analyzed these ratings in a 13 (emotions) $\times 2$ (individual vs. group level) repeated-measures ANOVA (Table 5 presents relevant means and standard deviations). Results revealed a significant emotions main effect, $F(12,1140)=59.59, p<.001, \eta^{2}=.39$. This indicates that (across individual and group levels) participants endorsed some emotions more strongly than others. The main effect of emotion level (individual vs. group) was marginal, $F(1,95)=$ $3.12, p=.081, \eta^{2}=.03$. On average, participants tended to endorse group-level emotions more strongly than individual emotions. More important, the main effects were qualified by a significant Emotions $\times$ Level interaction, $F(12,1140)=7.01, p<$ $.001, \eta^{2}=.07$. This means that there were significant differences between the profile of emotions that participants reported when thinking of themselves as individuals and as Americans. ${ }^{6}$ In this regard, it is noteworthy that, for nostalgia, the correlation between individual and group-level emotion was numerically smaller than for any other emotion and significantly smaller than for fear,

\footnotetext{
${ }^{5}$ Following recommendations by Singer (1998), we centered individual emotion ratings (a Level 1 predictor) about each participant's mean (participant-mean centered). We also mean-centered the average or prototypical group-level emotion ratings (a Level 1 predictor). By definition, the average or prototypical group-level emotion profile is identical for each participant, and in this case, grand-mean and participant-mean centering are mathematically equivalent.

${ }^{6}$ When we included age as a continuous between-subjects variable in the original 13 (emotions) $\times 2$ (individual vs. group level) repeated-measures ANOVA, we obtained a significant Emotions $\times$ Age interaction, $F(12$ $1116)=3.51, p<.001, \eta^{2}=.04$. Across individual- and group-level emotions, higher age was associated with a more positive (and less negative) emotion profile. A contrast comparing the six negative emotions versus the seven positive emotions (including nostalgia) revealed that positive emotions exceeded negative emotions by a wider margin among older ( 1 standard deviation above the mean), $F(1,93)=70.79, p<.001$, $\eta^{2}=.42$, than younger ( 1 standard deviation below the mean), $F(1,93)=$ $26.10, p<.001, \eta^{2}=.21$, adults. This finding is broadly consistent with research showing that as people get older, they become more successful at maintaining well-being and positive emotions (for a review, see Charles \& Carstensen, 2007).
} 
gratitude, respect, and hope $(z \mathrm{~s}>2.14, p \mathrm{~s}<.032$, using Dunn \& Clark's (1969) test for comparing dependent correlations with no variable in common; Table 5 , in boldface).

Group-level nostalgia is distinct from other group-level emotions. Group-level nostalgia was positively correlated with the other six positive group-level emotions (Table 5). These correlations remained statistically significant when controlling for individual nostalgia in a partial-correlation analysis, $r \mathrm{~s}(96) \geq .31$, $p s<.002$. Nonetheless, group-level nostalgia overlapped less with the other positive group-level emotions, $r \mathrm{~s}(96) \leq .61$, than these emotions overlapped with each other, $r \mathrm{~s}(96) \geq .64$. Correlations of group-level nostalgia with the six negative group-level emotions were numerically small and nonsignificant, $r \mathrm{~s}(96) \leq|.11|, p \mathrm{~s}>$ .276. These results attest to the discreteness of collective nostalgia.

\section{Discussion}

The key objective of Study 4 was to firmly establish collective nostalgia as a group-level emotion by assessing whether it is socially shared within groups (Criterion 4). As Smith et al. (2007) formulated this criterion in relation to the convergence of grouplevel emotions within larger and more abstract social categories (e.g., nationality), we operationalized collective nostalgia by instructing members of the U.S. general public to indicate to what extent they generally experienced nostalgia (and a number of additional positive and negative emotions) when they think of themselves as Americans. By so doing, we were able to assess the convergence of collective nostalgia among members of a larger, abstract social category and move beyond the more specific focus on shared experiences in face-to-face groups. Results provided clear evidence for convergence of group-level emotions-including nostalgia - toward the average or prototypical American emotion profile. Furthermore, by examining collective nostalgia in conjunction with other individual- and group-level emotions, Study 4 provided evidence that participants identified collective nostalgia as a discrete group-level emotion. Jointly, these findings indicate that an explicit focus on shared experiences (as adopted in the preceding studies) is sufficient but not necessary for experiencing collective nostalgia that is socially shared and distinct from personal nostalgia.

Although Study 4 was not designed to identify why collective nostalgia is socially shared, a possible explanation is that thinking about oneself as a group member triggers iconic memories of meaningful and endearing episodes in the history of the group (see Preliminary Investigation). For examples, many Americans, when they think of themselves in terms of their nationality, remember nostalgically the first moon landing in 1969, the Los Angeles Olympic Games of 1984, or the 2008 election of the first Black president in U.S. history. As a result of thinking about similar events, these individuals may experience similar levels of collective nostalgia or, as Smith et al. (2007) put it, "they are all responding to more or less the same events in more or less the same way" (p. 443). If this is the case, it would suggest that whereas an explicit focus on shared experiences is not necessary for collective nostalgia, an implicit common frame of reference is. This, in turn, carries the important implication that collective nostalgia may confer benefits even on larger, abstract groups and social categories, based on the recognition that other members, whom one may have never met, nonetheless experience the same feelings when contemplating certain iconic events in the life of the group or category. This is a fruitful direction for future research. A related question is whether collective nostalgia, when induced through group-based sharing of the past (e.g., older persons sharing memories of a bygone era; C. Haslam et al., 2010), entails benefits above and beyond those entailed by collective nostalgia, when induced via isolated personal reflection on the shared past.

\section{General Discussion}

Whereas previous work has documented various benefits that personal nostalgia confers on the individual (Routledge, Wildschut, Sedikides, \& Juhl, 2013; Sedikides, Wildschut, Arndt, \& Routledge, 2008), the current research begins to identify benefits that collective nostalgia confers on the group. It also constitutes a key step toward establishing collective nostalgia as a group-level emotion with important implications for group processes.

\section{Collective Nostalgia Bestows Benefits on the Ingroup}

In Study 1, we found that British undergraduates who brought to mind a nostalgic event that they had experienced together with ingroup members (collective nostalgia) evinced more positive ingroup evaluation and stronger action tendencies to approach (and not avoid) ingroup members than did participants who (a) recalled a nostalgic event from their personal life as a unique individual (personal nostalgia), (b) recalled a positive past event that they had experienced together with ingroup members (collective positive), and (c) did not recall a past event (no recall). The comparison to personal nostalgia was crucial, because it showed that collective nostalgia could be distinguished from the analogous individuallevel emotion. The comparison to a positive shared experience revealed that collective nostalgia bestows unique benefits on the group, above and beyond recalling positive collective events per se. This casts doubt on the possibility that the beneficial effects of collective nostalgia are due merely to PA and extends mounting evidence that personal nostalgia exerts unique beneficial effects above and beyond PA. The comparison to the no-recall control condition is meaningful, because it revealed that the impact of collective nostalgia was sufficiently strong to dislodge participants' natural or baseline opinion of the ingroup from its moorings.

In Study 2, also with British undergraduates, we demonstrated that collective (compared with personal) nostalgia increased tangible behavioral intentions to support the ingroup more so than did recalling an ordinary collective (compared with personal) event. This beneficial effect of collective nostalgia was mediated by CSE and, more specifically, the importance of ingroup membership to participants' self-concept. These results provided further corroborating evidence that collective nostalgia bestows unique benefits on the group and shed light on the precise psychological mechanism linking collective nostalgia to support intentions that entailed real sacrifices of time and effort. We also obtained evidence that recalling collective (compared with personal) experiences per se increases perceived positive regard for the ingroup by outsiders, which, in turn, increases support intentions. Still, attesting to its remarkable impact, the specific effect of collective nostalgia rose significantly above this more general effect of recalling collective (compared with personal) experiences.

Finally, in Study 3, we established that for Irish undergraduates who identified strongly with their national ingroup, recalling a 
nostalgic (compared with ordinary) event shared with ingroup members resulted in greater financial sacrifices to punish transgressions perpetrated against the ingroup. This extended the role of collective nostalgia into the domain of group behavior.

\section{Collective Nostalgia Is a Group-Level Emotion}

Our research provided evidence that nostalgia meets the four criteria specified by Smith et al. (2007) for identifying group-level emotions. Relevant to Criterion 1 (group-level emotions can be differentiated from analogous individual-level emotions), Studies 1 and 2 showed that collective nostalgia was more beneficial to the ingroup than was personal nostalgia. Consistent with Criterion 2 (group-level emotions motivate and regulate attitudes and behavior in relation to social groups), Studies 1-3 demonstrated that collective nostalgia conferred benefits on the ingroup, as manifested in positive ingroup evaluations and approach-oriented action tendencies (Study 1), behavioral intentions to support the ingroup (Study 2), and tangible behavior (Study 3). In accordance with Criterion 3 (the experience and expression of group-level emotions are shaped by social identification), Study 3 revealed that when social identification is high (compared with low), collective nostalgia has more far-reaching implications in terms of the financial sacrifices willingly incurred on behalf of the ingroup. Study 4 completed the puzzle by furnishing evidence for Criterion 4 (group-level emotions converge within a group): within a large, abstract social group (Americans), ratings of group-level nostalgia converged toward the group average, indicating that collective nostalgia is socially shared.

\section{Limitations and Future Directions}

Before generalizing from the findings, one must keep in mind that participants in our studies were predominantly young adult women from Western cultures. Studies 1, 2, and 4 included a sufficient number of male participants to allow adequate tests of gender differences ( $n \mathrm{~s}=96,47$, and 64 , respectively). No material gender differences emerged, providing evidence for the generalizability of our findings where gender is concerned. With regard to culture, we recruited British (Studies 1 and 2), Irish (Study 3), and U.S. (Study 4) participants and obtained coherent results across these three Western samples. This is further evidence for the generalizability of our findings, but it is nonetheless important for future research to examine collective nostalgia and its implications for group processes in non-Western cultures. Whereas social groups may be a more impactful source of self-definition in collectivistic cultures (e.g., China and Japan) than in individualistic cultures (e.g., United Kingdom and United States), a sense of belongingness to ingroups is pivotal in any culture (Baumeister \& Leary, 1995). Our working hypothesis, then, is that social identity processes operate in similar ways across cultures (Abrams, 2013; Sedikides, Gaertner, Luke, O’Mara, \& Gebauer, 2013). For example, social identification promotes organizational commitment in both individualistic and collectivistic cultures (de Moura et al., 2009). The question of whether our present findings generalize to collectivist cultures and other ingroups (e.g., familial, professional, ethnic) awaits empirical scrutiny. Turning to the role of age, with the exception of Study 4, our studies predominantly involved college-age participants. In Study 4, we recruited an online sample with a higher average age and a wider age range $\left(M_{\text {age }}=34.60\right.$ years, $S D_{\text {age }}=11.56$, range age $=19-64$ years). Consistent with the literature on emotion regulation across the life span (Charles \& Carstensen, 2007), older (compared with younger) participants generally showed a more positive emotion profile (footnote 6). We found no evidence, however, that age influenced the experience of collective nostalgia or the extent to which it was socially shared.

Clarifying the role of collective nostalgia across the life span is a priority for future research. Those conducting such research should study representative population samples that include participants in advanced old age (i.e., beyond the Study 4 age range). Socioemotional selectivity theory (Carstensen, Isaacowitz, \& Charles, 1999) proposes that with advancing age, people come to view their life span as limited and shift attention from futureoriented and knowledge-related goals toward a desire to find purpose and meaning in life, to enjoy intimate friendships, and to be embedded in a social network. This may mean that collective nostalgia acquires greater significance in advanced old age. When bereavement and physical frailty render older adults vulnerable to loneliness (Victor, Scambler, Bowling, \& Bond, 2005), collective nostalgia may serve to maintain a sense of belongingness and social identity (Cavanaugh, 1989; C. Haslam et al., 2010). Consistent with this possibility, recent findings indicate that proneness to nostalgia is more strongly related to psychological well-being among older (compared with younger) individuals (Hepper, Robertson, Wildschut, Sedikides, \& Routledge, 2014).

Although social connectedness may acquire special significance in old age, it is associated with increased psychological and physical health across the entire life span (Berkman, 1995). In Study 2 , collective nostalgia increased CSE. CSE, in turn, is associated with psychological well-being, even when the effects of personal selfesteem are controlled (Crocker, Luhtanen, Blaine, \& Broadnax, 1994). Future researchers should test whether collective nostalgia, by virtue of its capacity to strengthen CSE, promotes psychological well-being (S. A. Haslam, Jetten, Postmes, \& Haslam, 2009; Iyer \& Jetten, 2011) and whether these well-being benefits are contingent upon the quality (e.g., positive or negative, continuous or discontinuous) of the shared group experiences (Iyer \& Jetten, 2011; Jetten \& Hutchison, 2011). Another important implication is that by increasing CSE, collective nostalgia may promote optimism about the future of society. Cheung et al. (2013) demonstrated this process on the individual level of analysis. They showed that personal nostalgia increased self-esteem, which, in turn, fostered optimism regarding one's future. On the collective level of analysis, collective nostalgia could strengthen belief in the group's efficacy to (re)establish cherished values in society through collective action (van Zomeren, Leach, \& Spears, 2012). This would suggest that collective nostalgia is not only consequential for individuals in groups but also for society at large.

These questions could be productively explored within the context of research on migration and biculturalism (Benet-Martínez, Leu, Lee, \& Morris, 2002). Migration is reaching unprecedented levels. There were 213 million migrants in 2010 (World Bank, 2013), projected to reach 350 million by the year 2025 (United Nations, Statistics Division, 2002; United Nations, Department of Economic and Social Affairs, Population Division, 2006). Collective nostalgia may be particularly important for expatriates who perceive the intersection of their two cultural identities as dissociated rather than overlapping and as inherently incompatible 
rather than harmonious (Brown \& Humphreys, 2002; Volkan, 1999). These individuals are likely to experience more acculturative stress (Benet-Martínez \& Haritatos, 2005) and may evoke collective nostalgia to counter this particular psychological threat and maintain psychological equanimity (Sedikides et al., 2009).

\section{Coda}

Collective nostalgia bestows unique benefits on the group. It precipitates favorable ingroup evaluations and global action tendencies to approach (and not avoid) ingroup members. Collective nostalgia also strengthens CSE, which mediates specific behavioral intentions to support the ingroup. Among individuals who identify strongly with the ingroup, collective nostalgia increases concrete, costly behaviors to support the ingroup (by punishing transgressions against an ingroup member). As a truly group-level emotion, collective nostalgia is crucial to understanding group processes and promises to offer integrative insights across diverse areas of psychological inquiry.

\section{References}

Abrams, D. (2013). Social identification and group processes. In J. M. Levine (Ed.), Frontiers of social psychology: Group processes (pp. 268-295). New York, NY: Psychology Press.

Ajzen, I., \& Fishbein, M. (2005). The influence of attitudes on behavior. In D. Albarracín, B. T. Johnson, \& M. P. Zanna (Eds.), The handbook of attitudes (pp. 173-221). Mahwah, NJ: Erlbaum.

Baumeister, R. F., \& Leary, M. R. (1995). The need to belong: Desire for interpersonal attachments as a fundamental human motivation. Psychological Bulletin, 117, 497-529. doi:10.1037/0033-2909.117.3.497

Benet-Martínez, V., \& Haritatos, J. (2005). Bicultural identity integration (BII): Components and psychological antecedents. Journal of Personality, 73, 1015-1050. doi:10.1111/j.1467-6494.2005.00337.x

Benet-Martínez, V., Leu, J., Lee, F., \& Morris, M. (2002). Negotiating biculturalism: Cultural frame-switching in biculturals with "oppositional" vs. "compatible" cultural identities. Journal of Cross-Cultural Psychology, 33, 492-516. doi:10.1177/0022022102033005005

Berkman, L. F. (1995). The role of social relations in health promotion. Psychosomatic Medicine, 57, 245-254. doi:10.1097/00006842199505000-00006

Blader, S. L., \& Tyler, T. R. (2009). Testing and extending the group engagement model: Linkages between social identity, procedural justice, economic outcomes, and extrarole behavior. Journal of Applied Psychology, 94, 445-464. doi:10.1037/a0013935

Blum, M. (2000). Remaking the East German past: Ostalgie, identity, and material culture. Journal of Popular Culture, 34, 229-253. doi:10.1111/ j.0022-3840.2000.3403_229.x

Brown, A. D., \& Humphreys, M. (2002). Nostalgia and the narrativization of identity: A Turkish case study. British Journal of Management, 13, 141-159. doi:10.1111/14678551.00228

Bullock, J. G., Green, D. P., \& Ha, S. E. (2010). Yes, but what's the mechanism? (don't expect an easy answer). Journal of Personality and Social Psychology, 98, 550-558. doi:10.1037/a0018933

Carstensen, L. L., Isaacowitz, D. M., \& Charles, S. T. (1999). Taking time seriously: A theory of socioemotional selectivity. American Psychologist, 54, 165-181. doi:10.1037/0003-066X.54.3.165

Castano, E., Yzerbyt, V., Paladino, M. P., \& Sacchi, S. (2002). I belong, therefore, I exist: Ingroup identification, ingroup entitativity, and ingroup bias. Personality and Social Psychology Bulletin, 28, 135-143. doi: $10.1177 / 0146167202282001$

Castelnuovo-Tedesco, P. (1980). Reminiscence and nostalgia: The pleasure and pain of remembering. In S. I. Greenspan \& G. H. Pollack (Eds.), The course of life: Psychoanalytic contributions toward understanding per- sonality development. Vol. III: Adulthood and the aging process (pp. 104-118). Washington, DC: U.S. Government Printing Office.

Cavanaugh, J. C. (1989). I have this feeling about everyday memory aging. Educational Gerontology, 15, 597-605. doi:10.1080/038012 7890150604

Charles, S. T., \& Carstensen, L. L. (2007). Emotion regulation and aging. In J. J. Gross (Ed.), Handbook of emotion regulation (pp. 307-327). New York, NY: Guilford Press.

Cheung, W. Y., Wildschut, T., Sedikides, C., Hepper, E. G., Arndt, J., \& Vingerhoets, A. J. J. M. (2013). Back to the future: Nostalgia increases optimism. Personality and Social Psychology Bulletin, 39, 1484-1496.

Crocker, J., Luhtanen, R., Blaine, B., \& Broadnax, S. (1994). Collective self-esteem and psychological well-being among White, Black, and Asian college students. Personality and Social Psychology Bulletin, 20, 503-513. doi:10.1177/0146167294205007

de Moura, G. R., Abrams, D., Retter, C., Gunnarsdottir, S., \& Ando, K. (2009). Identification as an organizational anchor: How identification and job satisfaction combine to predict turnover intention. European Journal of Social Psychology, 39, 540-557. doi:10.1002/ejsp.553

Dimitriadou, M., Maciejowski, B., Wildschut, T., \& Sedikides, C. (2014). Everything is better in Ithaca: Collective nostalgia increases ethnocentric product preferences. Unpublished manuscript. Imperial College Business School, Imperial College London, London, England.

Dunn, O. J., \& Clark, V. A. (1969). Correlation coefficients measured on the same individuals. Journal of the American Statistical Association, 64, 366-377. doi:10.1080/01621459.1969.10500981

Edwards, J. R., \& Lambert, L. S. (2007). Methods for integrating moderation and mediation: A general analytical framework using moderated path analysis. Psychological Methods, 12, 1-22. doi:10.1037/1082989X.12.1.1

Ellemers, N., Kortekaas, P., \& Ouwerkerk, J. W. (1999). Selfcategorisation, commitment to the group and group self-esteem as related but distinct aspects of social identity. European Journal of Social Psychology, 29, 371-389.

Ellemers, N., Spears, R., \& Doosje, B. (2002). Self and social identity. Annual Review of Psychology, 53, 161-186. doi:10.1146/annurev.psych .53 .100901 .135228

Fazio, R. H., \& Towles-Schwen, T. (1999). The MODE model of attitudebehavior processes. In S. Chaiken \& Y. Trope (Eds.), Dual-process theories in social psychology (pp. 97-116). New York, NY: Guilford Press.

Fehr, E., \& Fischbacher, U. (2004). Third-party punishment and social norms. Evolution and Human Behavior, 25, 63-87. doi:10.1016/S10905138(04)00005-4

Fiedler, K., Schott, M., \& Meiser, T. (2011). What mediation analysis can (not) do. Journal of Experimental Social Psychology, 47, 1231-1236. doi:10.1016/j.jesp.2011.05.007

Fodor, N. (1950). Varieties of nostalgia. Psychoanalytic Review, 37, 2538.

Frijda, N. H. (1986). The emotions. New York, NY: Cambridge University Press.

Frost, I. (1938). Homesickness and immigrant psychoses. Journal of Mental Science, 84, 801-847.

Fuller, J. B., Hester, K., Barnett, T., Frey, L., Relyea, C., \& Beu, D. (2006). Perceived external prestige and internal respect: New insights into the organizational identification process. Human Relations, 59, 815-846. doi:10.1177/0018726706067148

Gabriel, Y. (1993). Organizational nostalgia: Reflections on "The Golden Age.” In S. Fineman (Ed.), Emotion in organizations (pp. 118-141). London, England: Sage.

Gaertner, L., Sedikides, C., \& Graetz, K. (1999). In search of selfdefinition: Motivational primacy of the individual self, motivational primacy of the collective self, or contextual primacy? Journal of Per- 
sonality and Social Psychology, 76, 5-18. doi:10.1037/0022-3514.76 .1 .5

Gherghina, S., \& Klymenko, L. (2012). Why look back? Citizens' attitudes toward the communist regime in Belarus, Russia, and Ukraine. Problems of Post-Communism, 59, 55-65. doi:10.2753/PPC1075-8216590105

Gramzow, R. H., \& Gaertner, L. (2005). Self-esteem and favoritism toward novel in-groups: The self as an evaluative base. Journal of Personality and Social Psychology, 88, 801-815. doi:10.1037/0022-3514.88.5.801

Gramzow, R. H., Gaertner, L., \& Sedikides, C. (2001). Memory for in-group and out-group information in a minimal group context: The self as an informational base. Journal of Personality and Social Psychology, 80, 188-205. doi:10.1037/0022-3514.80.2.188

Haslam, C., Haslam, S. A., Jetten, J., Bevins, A., Ravenscroft, S., \& Tonks, J. (2010). The social treatment: The benefits of group interventions in residential care settings. Psychology and Aging, 25, 157-167. doi: $10.1037 / \mathrm{a} 0018256$

Haslam, S. A., Jetten, J., Postmes, T., \& Haslam, C. (2009). Social identity, health and well-being: An emerging agenda for applied psychology. Applied Psychology, 58, 1-23. doi:10.1111/j.1464-0597.2008.00379.x

Hayes, A. F. (2013). Introduction to mediation, moderation, and conditional process analysis: A regression-based approach. New York, NY: Guilford Press.

Hepper, E. G., Ritchie, T. D., Sedikides, C., \& Wildschut, T. (2012). Odyssey's end: Lay conceptions of nostalgia reflect its original Homeric meaning. Emotion, 12, 102-119. doi:10.1037/a0025167

Hepper, E. G., Robertson, S., Wildschut, T., Sedikides, C., \& Routledge, C. (2014). Time capsule: Nostalgia shields psychological well-being from limited time horizons. Manuscript submitted for publication.

Hepper, E. G., Wildschut, T., Sedikides, C., Ritchie, T. D., Yung, Y.-F., Hansen, N., . . . Zhou, X. (2014). Pancultural nostalgia: Prototypical conceptions across cultures. Emotion, 14, 733-747. doi:10.1037/ a0036790

Holak, S. L., \& Havlena, W. J. (1998). Feelings, fantasies and memories: An examination of the emotional components of nostalgia. Journal of Business Research, 42, 217-226. doi:10.1016/S0148-2963(97)00119-7

Iyer, A., \& Jetten, J. (2011). What's left behind: Identity continuity moderates the effect of nostalgia on well-being and life choices. Journal of Personality and Social Psychology, 101, 94-108. doi:10.1037/ a0022496

Iyer, A., \& Leach, C. W. (2009). Emotion in inter-group relations. European Review of Social Psychology, 19, 86-125. doi:10.1080/ 10463280802079738

Jetten, J., \& Hutchison, P. (2011). When groups have a lot to lose: Historical continuity enhances resistance to a merger. European Journal of Social Psychology, 41, 335-343. doi:10.1002/ejsp.779

Kahn, J. H., Tobin, R. M., Massey, A. E., \& Anderson, J. A. (2007). Measuring emotional expression with the Linguistic Inquiry and Word Count. American Journal of Psychology, 120, 263-286.

Kim, W. B. (2010). Nostalgia, anxiety, and hope: Migration and ethnic identity of Chosonjok in China. Pacific Affairs, 83, 95-114. doi: $10.5509 / 201083195$

Lee, J. (1992). Comparison of variance between correlated samples. Computer Applications in the Biosciences, 8, 405-406. doi:10.1093/ bioinformatics/8.4.405

Luhtanen, R., \& Crocker, J. (1992). A collective self-esteem scale: Selfevaluation of one's social identity. Personality and Social Psychology Bulletin, 18, 302-318. doi:10.1177/0146167292183006

Mackie, D. M., Devos, T., \& Smith, E. R. (2000). Intergroup emotions: Explaining offensive action tendencies in an intergroup context. Journal of Personality and Social Psychology, 79, 602-610. doi:10.1037/00223514.79.4.602

Mackie, D. M., \& Smith, E. R. (1998). Intergroup relations: Insights from a theoretically integrative approach. Psychological Review, 105, 499529. doi:10.1037/0033-295X.105.3.499
McCoy, S. K., \& Major, B. (2003). Group identification moderates emotional responses to perceived prejudice. Personality and Social Psychology Bulletin, 29, 1005-1017. doi:10.1177/0146167203253466

Merchant, A., Ford, J. B., \& Rose, G. (2011). How personal nostalgia influences giving to charity. Journal of Business Research, 64, 610616. doi:10.1016/j.jbusres.2010.06.013

Milligan, M. J. (2003). Displacement and identity discontinuity: The role of nostalgia in establishing new identity categories. Symbolic Interaction, 26, 381-403. doi:10.1525/si.2003.26.3.381

Pennebaker, J. W., Chung, C. K., Ireland, M., Gonzalez, A., \& Booth, R. J. (2007). The development and psychometric properties of LIWC2007 [Manual for computer software]. Austin, TX: LIWC.net.

Pearsall, J. (1998). The new Oxford dictionary of English. Oxford, England: Oxford University Press.

Pitman, E. G. (1939). A note on normal correlation. Biometrika, 31, 9-12. doi:10.1093/biomet/31.1-2.9

Ramos, M. R., Correia, I., \& Alves, H. (2014). To believe or not believe in a just world? The psychological costs of threats to the belief in a just world and the role of attributions. Self and Identity, 13, 257-273. doi: $10.1080 / 15298868.2013 .798890$

Rosch, E. (1978). Principles of categorization. In E. Rosch \& B. B. Lloyd (Eds.), Cognition and categorization (pp. 27-71). Hillsdale, NJ: Erlbaum.

Routledge, C., Arndt, J., Sedikides, C., \& Wildschut, T. (2008). A blast from the past: The terror management function of nostalgia. Journal of Experimental Social Psychology, 44, 132-140. doi:10.1016/j.jesp.2006 .11 .001

Routledge, C., Arndt, J., Wildschut, T., Sedikides, C., Hart, C. M., Juhl, J., . . S Schlotz, W. (2011). The past makes the present meaningful: Nostalgia as an existential resource. Journal of Personality and Social Psychology, 101, 638-652. doi:10.1037/a0024292

Routledge, C., Wildschut, T., Sedikides, C., Arndt, J., \& Juhl, J. (2012). The power of the past: Nostalgia as a meaning-making resource. Memory, 20, 452-460. doi:10.1080/09658211.2012.677452

Routledge, C., Wildschut, T., Sedikides, C., \& Juhl, J. (2013). Nostalgia as a resource for psychological health and well-being. Social and Personality Psychology Compass, 7, 808-818. doi:10.1111/spc3.12070

Sedikides, C., Gaertner, L., Luke, M. A., O’Mara, E. M., \& Gebauer, J. (2013). A three-tier hierarchy of motivational self-potency: Individual self, relational self, collective self. Advances in Experimental Social Psychology, 48, 235-295. doi:10.1016/B978-0-12-407188-9.00005-3

Sedikides, C., Wildschut, T., Arndt, J., \& Routledge, C. (2008). Nostalgia: Past, present, and future. Current Directions in Psychological Science, 17, 304-307. doi:10.1111/j.1467-8721.2008.00595.x

Sedikides, C., Wildschut, T., Routledge, C., Arndt, J., \& Zhou, X. (2009). Buffering acculturative stress and facilitating cultural adaptation: Nostalgia as a psychological resource. In R. S. Wyer, C. Chiu, \& Y. Hong (Eds.), Understanding culture: Theory, research and application (pp. 361-378). New York, NY: Psychology Press.

Simmons, J. P., Nelson, L. D., \& Simonsohn, U. (2011). False-positive psychology: Undisclosed flexibility in data collection and analysis allows presenting anything as significant. Psychological Science, 22, 1359-1366. doi:10.1177/0956797611417632

Singer, J. (1998). Using SAS PROC MIXED to fit multilevel models, hierarchical models, and individual growth models. Journal of Educational and Behavioral Statistics, 24, 323-355. doi:10.2307/1165280

Smith, E. R. (1993). Social identity and social emotions: Toward new conceptualizations of prejudice. In D. M. Mackie \& D. L. Hamilton (Eds.), Affect, cognition, and stereotyping: Interactive processes in group perception (pp. 297-315). San Diego, CA: Academic Press. doi:10.1016/B978-0-08-088579-7.50017-X

Smith, E. R. (1999). Affective and cognitive implications of a group becoming part of the self: New models of prejudice and of the self- 
concept. In D. Abrams \& M. A. Hogg (Eds.), Social identity and social cognition (pp. 183-196). Oxford, England: Blackwell.

Smith, E. R., Seger, C. R., \& Mackie, D. M. (2007). Can emotions be truly group-level? Evidence regarding four conceptual criteria. Journal of Personality and Social Psychology, 93, 431-446. doi:10.1037/00223514.93.3.431

Stephan, E., Sedikides, C., \& Wildschut, T. (2012). Mental travel into the past: Differentiating recollections of nostalgic, ordinary, and positive events. European Journal of Social Psychology, 42, 290-298. doi: 10.1002/ejsp. 1865

Stephan, E., Wildschut, T., Sedikides, C., Zhou, X., He, W., Routledge, C., . . Vingerhoets, A. J. J. M. (2014). The mnemonic mover: Nostalgia regulates avoidance and approach motivation. Emotion, 14, 545-561. doi: $10.1037 / \mathrm{a} 0035673$

Tarrant, M., North, A. C., \& Hargreaves, D. J. (2004). Adolescents' intergroup attributions: A comparison of two social identities. Journal of Youth and Adolescence, 33, 177-185. doi:10.1023/B:JOYO.0000 025317.96224 .75

Tropp, L. R., \& Wright, S. C. (2001). Ingroup identification as the inclusion of ingroup in the self. Personality and Social Psychology Bulletin, 27, 585-600. doi:10.1177/0146167201275007

Turner, R. N., Wildschut, T., \& Sedikides, C. (2012). Dropping the weight stigma: Nostalgia improves attitudes toward persons who are overweight. Journal of Experimental Social Psychology, 48, 130-137. doi: 10.1016/j.jesp.2011.09.007

Turner, R. N., Wildschut, T., Sedikides, C., \& Gheorghiu, M. (2013). Combating the mental health stigma with nostalgia. European Journal of Social Psychology, 43, 413-422. doi:10.1002/ejsp.1952

Tyler, T. R., \& Blader, S. L. (2003). The group engagement model: Procedural justice, social identity, and cooperative behavior. Personality and Social Psychology Review, 7, 349-361. doi:10.1207/S15327957PSPR0704_07

United Nations, Statistics Division. (2002). UN population report 2002. New York, NY: Author.

United Nations, Department of Economic and Social Affairs, Population Division. (2006). Trends in the total migrant stock: The 2005 revision. CD-ROM Documentation. (Report No. POP/DB/MIG/Rev.2005/Doc). New York, NY: Author.

van Tilburg, W. A. P., \& Igou, E. R. (2011). On boredom and social identity: A pragmatic meaning-regulation approach. Personality and Social Psychology Bulletin, 37, 1679-1691. doi:10.1177/0146167211418530 van Tilburg, W. A. P., Igou, E. R., \& Sedikides, C. (2013). In search of meaningfulness: Nostalgia as an antidote to boredom. Emotion, 13, 450-461. doi:10.1037/a0030442

van Zomeren, M., Leach, C. W., \& Spears, R. (2012). Protesters as "passionate economists": A dynamic dual pathway model of approach coping with collective disadvantage. Personality and Social Psychology Review, 16, 180-198. doi:10.1177/1088868311430835

Velikonja, M. (2009). Lost in transition: Nostalgia for socialism in postsocialist countries. East European Politics and Societies, 23, 535-551. doi: $10.1177 / 0888325409345140$

Verplanken, B. (2012). When bittersweet turns sour: Adverse effects of nostalgia on habitual worriers. European Journal of Social Psychology, 42, 285-289. doi:10.1002/ejsp. 1852

Vess, M., Arndt, J., Routledge, C., Sedikides, C., \& Wildschut, T. (2012) Nostalgia as a resource for the self. Self and Identity, 11, 273-284 doi:10.1080/15298868.2010.521452

Victor, C. A., Scambler, S. J., Bowling, A., \& Bond, J. (2005). The prevalence of, and risk factors for, loneliness in later life: A survey of older people in Great Britain. Ageing \& Society, 25, 357-375. doi: 10.1017/S0144686X04003332

Volkan, V. D. (1999). Nostalgia as a linking phenomenon. Journal of Applied Psychoanalytic Studies, 1, 169-179. doi:10.1023/A: 1023037222314

Wildschut, T., Sedikides, C., Arndt, J., \& Routledge, C. (2006). Nostalgia: Content, triggers, functions. Journal of Personality and Social Psychology, 91, 975-993. doi:10.1037/0022-3514.91.5.975

Wildschut, T., Sedikides, C., Routledge, C., Arndt, J., \& Cordaro, F. (2010). Nostalgia as a repository of social connectedness: The role of attachment-related avoidance. Journal of Personality and Social Psychology, 98, 573-586. doi:10.1037/a0017597

World Bank. (2013). World DataBank. Retrieved from http://databank .worldbank.org/data/

Zhou, X., Sedikides, C., Wildschut, T., \& Gao, D. (2008). Counteracting loneliness: On the restorative function of nostalgia. Psychological Science, 19, 1023-1029. doi:10.1111/j.1467-9280.2008.02194.x

Zhou, X., Wildschut, T., Sedikides, C., Chen, X., \& Vingerhoets, A. J. J. M. (2012). Heartwarming memories: Nostalgia maintains physiological comfort. Emotion, 12, 678-684. doi:10.1037/a0027236

Zhou, X., Wildschut, T., Sedikides, C., Shi, K., \& Feng, C. (2012). Nostalgia: The gift that keeps on giving. Journal of Consumer Research, 39, 39-50, doi:10.1086/662199 


\section{Appendix}

\section{Recall Instructions in Study 1}

\section{Collective Nostalgia}

According to the Oxford Dictionary, nostalgia is defined as a "sentimental longing for the past." Please bring to mind a nostalgic event that you experienced in your student life at the University of Southampton. This should be a nostalgic event that involves other University of Southampton students. Specifically, try to think of a past event that you shared with other University of Southampton students that makes you feel particularly nostalgic.

\section{Personal Nostalgia}

According to the Oxford Dictionary, nostalgia is defined as a "sentimental longing for the past." Please bring to mind a nostalgic event that you experienced in your personal life. This should be a nostalgic event that is about you personally and your life as a unique individual. Specifically, try to think of a past event in your personal life that makes you feel particularly nostalgic.

\section{Positive Collective Event}

Please bring to mind a lucky event that you experienced in your student life at the University of Southampton. This should be a lucky event that involves other University of Southampton students. Specifically, try to think of a positive past event in your student life that you shared with other University of Southampton students that was brought on by chance rather than through your own actions (e.g., you unexpectedly found a lost item).

Received July 5, 2013

Revision received June 26, 2014

Accepted July 17, 2014

\section{E-Mail Notification of Your Latest Issue Online!}

Would you like to know when the next issue of your favorite APA journal will be available online? This service is now available to you. Sign up at http://notify.apa.org/ and you will be notified by e-mail when issues of interest to you become available! 